\title{
SUR LES ÉQUATIONS DIFFÉRENTIELLES DU SECOND ORDRE ET DU PREMIER DEGRÉ DONT LINTÉGRALE GÉNÉRALE EST A POINTS CRITIQUES FIXES.
}

PAR

\author{
B. GAMBIER \\ à Moxpreitler.
}

\section{Introduction.}

1. - Objet de ce mémoire. - M. Painleví a indiqué une méthode pour déterminer toutes les équations diférentielles (algébriques) du second ordre dont les points critiques sont fixes et il a développé cette methode explicitement dans le cas où l'équation est de la forme

$$
y^{\prime \prime}=R\left(y^{\prime}, y, x\right)
$$

$R$ rationnel en $y^{\prime}$, algébrique en $y$, algébrique ou analytique en $x$.

Un mémoire publié dans les Acta Mathematica (1902) renferme le tableau de toutes les équations (I) à points critiques fixes.

Ayant commencé, sur les conseils de M. Painlevé, l'application de sa méthode aux équations qui sont non plus du premier degré mais du second en $y^{\prime \prime}$, j'ai été conduit à réviser l'énumération des équations (I) et j'ai constaté que les tableaux dressés par M. Painlevé présentaient une lacune que je comblerai dans le présent mémoire. Cette lacune provient de ce que dans la discussion des cas nombreux que la méthode conduit à distinguer, M. PaInLevé en a laissé échapper un, qui se trouve correspondre à des types nombreux et importants. Je préciserai d'abord cette omission. 
2. On peut toujours mettre l'équation sous la forme

$$
y^{\prime \prime}=\varrho\left(y^{\prime}, y, u, x\right)
$$

$\varrho$ étant rationnel en $y^{\prime}, y, u$ et $u$ étant lié à $y$ par une relation algébrique qui dépend analytiquement de $x$ soit

$$
H(u, y, x)=\text { o. }
$$

M. Painlevé montre d'abord que pour $x$ arbitraire la relation (3) entre $y$ et $u$ doit être de genre o ou I. Dans le cas où elle est de genre I les tableaux de M. Painleví sont complets: je n'y reviendrai plus. Dans le cas où elle est de genre zéro on peut toujours moyennant un changement de variable très simple supposer que $R\left(y^{\prime}, y, x\right)$ est rationnel en $y^{\prime}$ et $y$. Dans ce cas, je vais indiquer pourquoi les tableaux de M. Painleví doivent être notablement complétés.

M. Painlevé établit d'abord que l'équation (I) est de la forme

$$
y^{\prime \prime}=A(y, x) y^{\prime 2}+B(y, x) y^{\prime}+C(y, x),
$$

où $A(y, x)$ est l'une des 9 expressions $\theta$

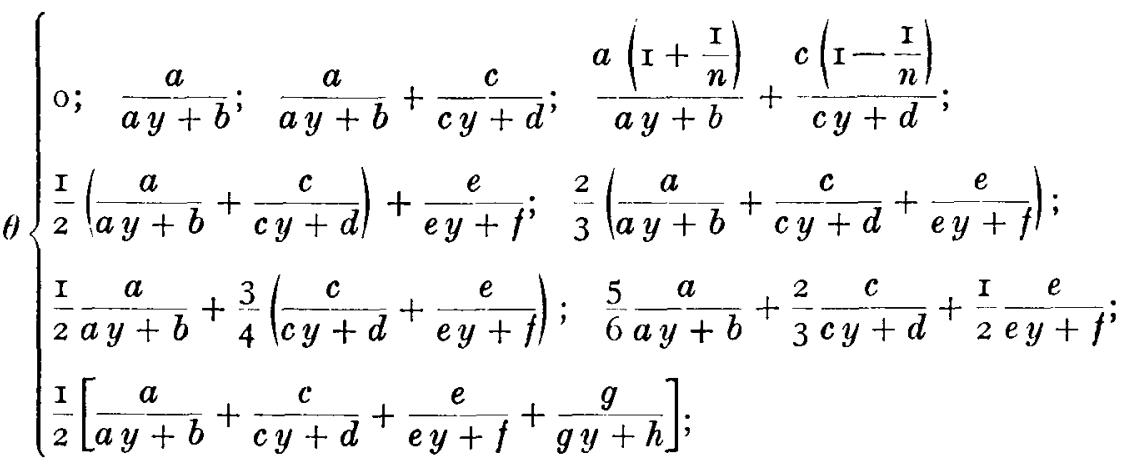

$a, b, \ldots, h$, désignent des fonctions de $x$ qui peuvent être identiquement nulles ou se réduire à des constantes, $n$ désigne un entier positif supérieur à I. En effectuant sur $y$ la transformation homographique $Y=y$ ou $Y=\frac{\mathrm{I}}{a y+b}$ on $Y=\frac{c y+d}{a y+b}$ ou $Y=\frac{(a f-b e)}{(c f-d e)} \frac{(c y+d)}{(a y+b)}$ suivant que $A$ coïncide avec la $I^{\grave{e} r e}$ de ces expressions, ou la seconde, ou avec la $3^{\text {ème }}$ et $4^{\text {ème }}$, ou enfin avec l'une quelconque des suivantes, on est ramené à une équation analogue

$$
Y^{\prime \prime}=A(Y, X) Y^{\prime 2}+B(Y, X) Y^{\prime}+C(Y, X) \text {, }
$$

où $A, B, C$ sont rationnels en $Y$ et où $A$ coincide avec une des 8 expressions $\theta^{\prime}$ 


$$
\theta^{\prime}\left\{\begin{array}{l}
0 ; \quad \frac{\mathrm{I}}{Y} ; \frac{\mathrm{I}-\frac{\mathrm{I}}{n}}{Y} ; n \text { entier }>\mathrm{I} ; \frac{\mathrm{I}}{2}\left(\frac{\mathrm{I}}{Y}+\frac{\mathrm{I}}{Y-\mathrm{I}}\right) ; \\
\frac{2}{3}\left(\frac{\mathrm{I}}{Y}+\frac{\mathrm{I}}{Y-\mathrm{I}}\right) ; \frac{3}{4}\left(\frac{\mathrm{I}}{Y}+\frac{\mathrm{I}}{Y-\mathrm{I}}\right) ; \frac{2}{3 Y}+\frac{\mathrm{I}}{2} \frac{\mathrm{I}}{Y-\mathrm{I}} ; \frac{\mathrm{I}}{2}\left(\frac{\mathrm{I}}{Y}+\frac{\mathrm{I}}{Y-\mathrm{I}}+\frac{\mathrm{I}}{Y-H}\right)
\end{array}\right.
$$

$H$ désignant soit une constante numérique c soit $X$.

M. Painlevé étudie ensuite les équations correspondant à chacune de ces 8 formes de $\boldsymbol{A}$. Or l'énumération de M. Painlevé est complète pour les 2 formes $A \equiv 0$ et $A \equiv \frac{\mathrm{I}}{Y}$ mais le cas $A \equiv \frac{\mathrm{I}-\frac{\mathrm{I}}{n}}{Y}$ présente une lacune importante et comme la discussion relative $\grave{a}$ ce cas retentit sur la discussion des 5 derniers, cette lacune entraîne l'existence de lacunes correspondantes dans les 5 derniers tableaux.

3. Ce n'est donc pas une erreur dans la méthode mais une omission dans l'application de la méthode qui explique les lacunes des tableaux de M. PaINLEVÉ et c'est par l'application même de cette méthode que j'ai comblé ces lacunes.

Cette méthode se décompose en deux méthodes distinctes:

$I^{0}$ une méthode qui fournit un certain ensemble de conditions nécessaires pour que l'équation ait ses points critiques fixes.

$2^{0}$ une méthode qui démontre que ces conditions sont suffisantes.

L'application de la méthode aux cas omis par M. Painlevé n'était pas toutefois sans présenter des difficultés. Tout d'abord des complications de calcul, si les conditions étaient formées maladroitement. Ensuite il n'était pas certain que les conditions ainsi obtenues fussent intégrables, c'est-à-dire qu'on pût écrire explicitement à l'aide d'un certain nombre de fonctions de $x$ arbitraires toutes les équations cherchées. Enfin parmi les équations obtenues, les unes sont intégrables, j'entends réductibles aux équations linéaires et aux quadratures, les autres sont irréductibles. Il fallait distinguer ces deux classes et notamment intégrer les équations intégrables, ce qui, comme on le verra plus loin, était pour certaines d'entre elles assez malaisé.

Je résume d'abord les résultats de cette discussion minutieuse. Les plus importants concernent les équations irréductibles, qui engendrent des transcendantes nouvelles.

4. Transcendentes nouvelles engendrées par les équations différentielles du second ordre. 
B. Gambier.

Il existe 6 types et 6 seulement d'équations différentielles (I) à points critiques fixes qui sont irréductibles au sens le plus rigoureux du terme (sens de M. DRACH). Ces 6 types peuvent recevoir les formes canoniques qui suivent $[\alpha, \beta, \gamma, \delta$ désignent des constantes numériques]

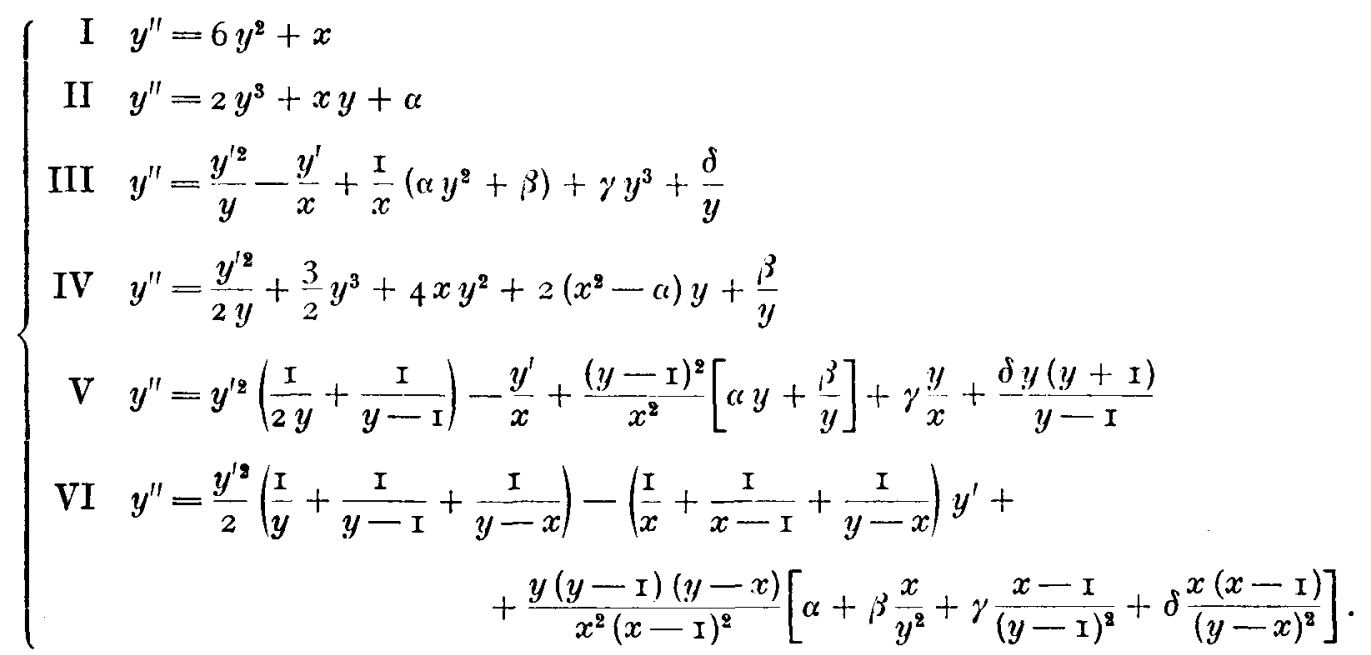

Par suite de l'omission signalée, M. Palnlevé n'avait obtenu que 3 types distincts d'équations irréductibles, à savoir les 3 premiers. Les tableaux qu'il avait formés ne contenaient pas l'équation IV; ils contenaient l'équation $V$ dans le cas où $\alpha=\beta=0$, auquel cas la transformation $y=\left(\frac{z+I}{z-I}\right)^{2}$ permet de la ramener au type III, tandis que si $\alpha, \beta$ ne sont pas nuls tous deux elle est irréductible au type III; de même l'équation VI ne figurait dans les tableaux de M. PaInLevé que dans le cas où $\alpha=\beta=\gamma=0, \delta=\frac{I}{2}$ auquel cas elle est intégrable, tandis que dans le cas général elle est irréductible.

L'intégrale générale $y(x)$ des équations I, II, IV est uniforme; celle des équations III et $V$ admet $x=0$ et $x=\infty$ comme points critiques transcendants; si l'on effectue, pour ces 2 types la transformation $x=e^{X}$, la fonction $y(X)$ est une transcendante uniforme. Enfin l'intégrale générale $y(x)$ de l'équation VI admet les points critiques transcendants $x=0, x=\mathrm{I}, x=\infty$.

5. La méthode employée par M. Painlevé pour montrer que l'équation $y^{\prime \prime}=6 y^{2}+x$ a ses points critiques fixes [Bulletin des Sciences Math. 1900] s'applique d'elle même aux autres équations. Dans une note des Comptes Rendus, 24 Décembre 1906, M. Painlevé a fait lui-même cette extension. Il a de plus montré que le type VI contient comme dégénérescences les 5 premiers: 
Équations différentielles dont l'intégrale générale est à points critiques fixes.

$\mathrm{V}$ est dégénérescence de VI; III et IV dégénérescences de V; II dégénérescence de III ou IV et enfin I est dégénérescence de II.

M. Painlevé l'établit de la façon suivante: faisons dans VI $\delta=\frac{\delta_{1}}{\varepsilon^{2}}$, $y=-\frac{\delta_{1}}{\varepsilon^{2}}+\frac{\gamma_{1}}{\varepsilon}, x=\mathrm{I}+\varepsilon X, y=Y$ d'où une équation

$$
Y^{\prime \prime}=Y^{\prime 2}\left(\frac{\mathrm{I}}{2 Y}+\frac{\mathrm{I}}{Y-\mathrm{I}}\right)-\frac{Y^{\prime}}{X}+\frac{(Y-\mathrm{I})^{2}}{X^{2}}\left[\alpha Y+\frac{\beta}{Y}\right]+\gamma \frac{Y}{X}+\frac{\delta Y(Y+\mathrm{I})}{Y-\mathrm{I}}+\varepsilon(\cdots)
$$

qui a ses points critiques fixes en même temps que $\mathrm{Vl}$ pour toute valeur numérique non nulle donnée à $\varepsilon$, et par suite encore pour $\varepsilon=0$ : or elle se réduit à $\mathrm{V}$ pour $\varepsilon=0$. L'application du même principe donne en faisant dans $\mathrm{V}$

$$
\beta=-\frac{\beta_{1}}{\varepsilon^{2}}, \quad \alpha=\frac{\beta_{1}}{\varepsilon^{2}}+\frac{\alpha_{1}}{\varepsilon}, \quad \gamma=\gamma_{1} \varepsilon, \quad \delta=\delta_{1} \varepsilon^{2}, \quad y=\mathrm{I}+\varepsilon Y, \quad x=X
$$

la dégénérescence

$$
Y^{\prime \prime}=\frac{Y^{\prime 2}}{Y}-\frac{Y^{\prime}}{X}+\frac{Y^{2}}{X^{2}}\left(\alpha_{1}+2 \beta_{1} Y\right)+\frac{\gamma_{1}}{X}+\frac{2 \delta_{1}}{Y}
$$

qui donne III en posant $X=\xi^{\mathrm{a}}, Y=r, \xi$

De même si dans $\mathrm{V}$ on fait

$$
\alpha=\frac{\mathrm{I}}{2 \varepsilon^{4}}, \quad \gamma=\frac{-\mathrm{I}}{\varepsilon^{4}}, \quad \delta=-\left(\frac{\mathrm{I}}{2 \varepsilon^{4}}+\frac{\delta_{1}}{\varepsilon^{2}}\right), y=\varepsilon Y, x=\mathrm{I}+\varepsilon X
$$

on a la dégénérescence

$$
Y^{\prime \prime}=\frac{Y^{\prime 2}}{2 Y}+\frac{3}{2} Y^{3}+2 X Y^{2}+Y\left(\frac{X^{2}}{2}+\delta_{1}\right)+\frac{\beta^{2}}{Y}
$$

qui donne IV en posant $X=\xi \sqrt{2}, r=Y \sqrt{2}$.

On pose maintenant dans III

$$
\begin{aligned}
& \gamma=-\delta=\frac{\mathrm{I}}{4 \varepsilon^{6}}, \quad \alpha=\frac{-\mathrm{I}}{2 \varepsilon^{6}}, \quad \beta=\frac{\mathrm{I}}{2 \varepsilon^{6}}\left[\mathrm{I}+4 \beta_{1} \varepsilon^{3}\right] \\
& x=\mathrm{I}+\varepsilon^{2} X, \quad y=\mathrm{I}+2 \varepsilon Y
\end{aligned}
$$

on a la dégénérescence $Y^{\prime \prime}=2 Y^{3}+X Y+\beta_{1}$ ou II.

De même dans IV

$$
\beta=-\frac{\mathrm{I}}{2 \varepsilon^{12}}, \alpha=-\frac{\mathrm{I}}{2 \varepsilon^{6}}-\alpha_{1}, \quad y=\frac{\mathrm{I}}{\varepsilon^{3}}\left(\mathrm{I}+2 \varepsilon^{2} Y\right), \quad x=\varepsilon X-\frac{\mathrm{I}}{\varepsilon^{3}}
$$

on a $Y^{\prime \prime}=8 Y^{3}+{ }_{4} X Y+\alpha_{1}$ et en posant $2 X=\xi \sqrt[s]{2}, r=Y \sqrt[3]{2}$ on a le type II. 
Enfin la substitution $y=\varepsilon Y+\frac{I}{\varepsilon^{5}}, \quad x=\varepsilon^{2} X-\frac{6}{\varepsilon^{10}}, \quad \alpha=\frac{4}{\varepsilon^{15}}$ transforme II en $Y^{\prime \prime}=6 Y^{2}+X+\varepsilon^{6}\left(2 Y^{3}+X Y\right)$ ce qui conduit bien à $\mathrm{I}$ comme dégénérescence.

Or M. Patnlevé a montré aux Comptes Rendus que l'équation $y^{\prime \prime}=6 y^{2}+x$ est irréductible au sens le plus rigoureux du terme (sens de M. DraCH). Il en résulte que les 5 suivantes sont aussi absolument irréductibles sauf peut-être pour des valeurs exceptionnelles des constantes $\alpha, \beta, \gamma, \delta$.

6. Formation des conditions nécessaires pour que l'équation (I) ait ses points critiques fixes.

Je voudrais indiquer maintenant la façon précise dont j'ai appliqué la méthode de M. Painleví pour former les conditions nécessaires que vérifie toute équation (I) à points critiques fixes. L'équation à étudier est de la forme

$$
y^{\prime \prime}=A(y, x) y^{\prime 2}+B(y, x) y^{\prime}+C(y, x)
$$

où $A$ est l'une des expressions $\theta^{\prime}$. Les expressions $B$ et $C$ sont des fonctions rationnelles en $y$, dont les pôles, d'après un résultat de M. PaInleví, sont simples et coincident avec les pôles de $A$; la transformation y $Y=$ r limite le degré de $B$ et $C$ en $y$ par l'application de ce même résultat au pôle $Y=0$.

J'emploie alors la substitution de M. PAINLejvé: $x=x_{0}+a X, Y=\alpha y$ d'où une équation dont l'intégrale générale est à points critiques fixes quelque soit $\alpha$, sauf peut-être pour $\alpha=0$, donc encore pour $\alpha=0$ : cette équation où $\alpha$ a été annulé, ou équation simplifiée est du type suivant

$$
\begin{aligned}
& y^{\prime \prime}=a_{0} y y^{\prime}+b_{0} y^{3} \quad \text { si } \quad A \equiv 0 \\
& y^{\prime \prime}=\frac{y^{\prime 2}}{y}+a_{0} y y^{\prime}+b_{0} y^{3} \quad \text { si } \quad A \equiv \frac{\mathrm{r}}{y}
\end{aligned}
$$

$$
y^{\prime \prime}=\frac{y^{\prime 2}}{y}\left(\mathrm{I}-\frac{\mathrm{I}}{n}\right)+a_{0} y y^{\prime}+b_{0} y^{3} \text { pour les } 6 \text { dernières formes de } A
$$

( $n$ entier $\geqslant 0$, différent de o ou de $\pm \mathrm{I} ; a_{0}, b_{0}$ désignent les valeurs pour $x=x_{0}$ de deux fonctions $a(x), b(x)$ calculées rationnellement au moyen des coefficients de l'équation proposée.)

C'est dans l'étude de la simplifiée (8) que M. Painlevé a laissé échapper un cas important; cela explique comment les résultats de M. PaInlevé étaient complets pour les 2 premières formes de $A$; la simplifiée (8) rentre dans les équations où $A$ a la $3^{\text {ème }}$ forme possible, et on voit comment les 5 dernières formes de $A$ conduisant à la même forme simplifiée, une telle omission pour la 
Équations différentielles dont l'intégrale générale est à points critiques fixes.

$3^{\text {d̀me }}$ forme de $A$ entraîne des omissions correspondantes pour les formes suivantes de $A$.

En tous les cas les 3 simplifiées s'étudient de la même façon: la variable indépendante $x$ manque, d'où résulte que si l'intégrale générale de cette simplifiée est a points critiques fixes, elle doit être de plus uniforme. Cette intégrale s'obtient par quadratures: si on écrit $y^{\prime}=u y^{2}, u$ vérifie une équation $u^{\prime \prime}=u^{\prime 2} f(u)$ où $f$ est rationnelle en $u$ et on a en même temps $y=r\left(u, u^{\prime}\right)$, $p$ étant rationnelle en $u$ et $u^{\prime}$ : or Briot et Bovquet ont déterminé toutes les formes possibles que peut avoir la fraction $f(u)$. L'application de leurs résultats donne ou bien $a(x) \equiv b(x) \equiv 0$ ou bien $a a^{2}+\beta b \equiv 0$, $a$ et $\beta$ étant deux constantes numériques non nulles toutes deux.

Occupons-nous d'abord de la seconde hypothèse $a a^{2}+\beta b \equiv 0 ; a(x), b(x)$ ne sont pas nuls tous 2 identiquement. Cherchons s'il n'existe pas, pour une valeur quelconque $x_{0}$ attribuée à $x$, une intégrale de l'équation, non simplifiée, qui est étudiée

$$
y^{n}=A(y, x) y^{\prime 2}+B(y, x) y^{\prime}+C(y, x)
$$

qui admette $x=x_{0}$ comme pôle. Si cela a lieu cette intégrale est représentée par un développement

$$
y=\frac{a_{1}}{\left(x-x_{0}\right)^{i}}+\frac{a_{2}}{\left(x-x_{0}\right)^{i}-1}+\cdots
$$

où $a_{1}, a_{2}, \ldots$ sont des constantes, $i$ un entier positif. En substituant dans l'équation (5) on doit avoir une identité; les termes polaires de degré le plus éleré sont respectivement

$$
\begin{array}{cccc}
y^{n} & \frac{y^{\prime 2}}{y} & y y^{\prime} & y^{3} \\
i+2 & i+2 & 2 i+\mathrm{I} & 3 i
\end{array}
$$

Il faut nécessairement que $i=\mathrm{I}$ sinon $i+2<2 i+\mathrm{I}<3 i$ sans égalité: si $b=0$ le terme de degré $3 i$ si $i>\mathrm{r}$ ne pourrait disparaître; si $b \equiv 0$, on aurait $a=0$ et alors le terme de degré $2 i+\mathrm{I}$ ne pourrait disparaître.

Nous faisons $i=\mathrm{I}$ et substituons: $a_{1}$ est donné par une équation du premier degré si $b \equiv 0$, du second si $b=0$; puis $a_{2}, a_{3}, \ldots$ s'expriment tous au moyen des coefficients précédents par une équation du premier degré, sauf peutêtre l'un d'eux et un seulement qui disparaît de l'équation qui devrait le fournir: mais alors il reste une relation qui est nécessaire pour que ce pôle existe: cette relation devant être vérifiée quel que soit $x_{0}$ entraîne une identité entre les coefficients de l'équation (5). 
Si l'on prend la première hypothèse $a(x) \equiv b(x) \equiv 0$, on arrive d̀ un résultat semblable. Je forme dans ce cas une nouvelle simplifiée en posant dans (5) $x=x_{0}+\alpha X, Y=\alpha^{2} y$, d'où par le même mécanisme

(6 bis) $\quad y^{\prime \prime}=e_{0} y^{2} \quad$ si $A \equiv 0$

(7 bis) $\quad y^{\prime \prime}=\frac{y^{\prime 2}}{y}+e_{0} y^{2} \quad$ si $A \equiv \frac{\mathrm{I}}{y}$

(8 bis) $\quad y^{\prime \prime}=\frac{y^{\prime 2}}{y}\left(\mathrm{I}-\frac{\mathrm{I}}{n}\right)+e_{0} y^{2}$ pour les 6 dernières formes de $A$

( $n$ désigne le même entier pour (8) et $(8 \mathrm{bis})$, $e_{0}$ est la valeur pour $x=x_{0}$ d'une fonction $e(x)$ calculée rationnellement au moyen des coefficients de l'équation 5 ).

Cette nouvelle simplifiée s'intègre encore par quadrature. J'emploie d'ailleurs l'artifice suivent, je pose $y^{\prime}=z y$ d'où $y=f\left(z, z^{\prime}\right)$ où $f$ est rationnel en $z$ et $z^{\prime}$ et l'équation

$$
z^{\prime \prime}=\gamma z z^{\prime}+\delta z^{3}
$$

où $\gamma$ et $\delta$ sont des constantes numériques: cette équation (Io) est précisément du type de la simplifiée (6) dont on a déterminé toutes les formes possibles. La conclusion est celle-ci: dans (6 bis) et $(7$ bis), $e(x)$ peut être identiquement nul ou non; dans ( $8 \mathrm{bis}$ ), $e(x)$ est identiquement nul, si $n$ n'est égal ni à 2 , ni à 4 , ni à -4 ; pour ces dernières valeurs de $n, e(x)$ peut être ou non identiquement nul.

Si $e(x) \equiv 0$ l'étude est finie.

Si $e(x)=\equiv$ o nous remontons comme plus haut de la simplifiée à l'équation (5): je cherche comme plus haut, si pour $x=x_{0}$ où $x_{0}$ est quelconque, s'il existe une intégrale admettant $x_{0}$ pour pôle. Pour des raisons semblables $i=2, a_{1}$ est donné par une équation du premier degré; les coefficients successifs se calculent comme plus haut et on arrive par le même mécanisme à une identité entre les coefficients de (5).

Cela fait on a épuisé tout ce que cette méthode peut donner pour la valeur $y=\infty$; il reste à faire, suivant la forme de $A$ une étude semblable pour les autres valeurs singulières de $y^{\prime \prime}$ considérées comme fonction de $y$, à savoir $y=0, \mathrm{r}$, ou $x$. On ramène cette étude à celle de $y=\infty$ par une substitution faite $\operatorname{sur}(5)$

$$
Y=\frac{\mathrm{I}}{y} \quad \text { ou } \quad Y=\frac{\mathrm{I}}{y-\mathrm{I}} \quad \text { ou } \quad Y=\frac{\mathrm{I}}{y-x} .
$$


Équations différentielles dont l'intégrale générale est à points critiques fixes.

7. Une question se pose ici: j’ai exprimé que les équations étudiées admettent des pôles mobiles (ou des zéros, ou des unités mobiles). Mais ces conditions sont-elles nécessaires pour que l'équation ait ses points critiques fixes?

La réponse est affirmative. M. PainLevé a donné l'énoncé général de ce théorème et l'a démontré en détail dans le cas où $A \equiv$ (Bulletin des Sciences mathématiques 1900). J'en donne une démonstration explicite pour l'un des cas nouveaux que je propose (voir plus loin, page 49). La démonstration employée montre qu'elle s'étend aux autres cas. Je supposerai done ce point acquis.

Rendons nous compte du nombre de conditions obtenues: il suffit de raisonner pour $y=\infty$; il y avait 3 cas à considérer, d'après la nature des fonctions $a(x), b(x), e(x)$.

$\left.\mathrm{I}^{\circ}\right) \quad \alpha a^{2}+\beta b \equiv 0$, sans que $a$ et $b$ soient identiquement nuls tous 2 .

Suivant que $a$ est nul ou non, il y a une ou deux familles de pôles mobiles simples, et comme une famille de pôles mobiles peut ou non conduire à une identité entre les coefficients de (5), nous avons en comptant $a a^{2}+\beta b=0$ au plus 3 relations identiquement vérifiées.

$\left.2^{0}\right) \quad a(x) \equiv b(x) \equiv 0, e(x) \equiv 0$, une famille de pôles doubles mobiles, donc comprenant $a(x) \equiv 0, b(x) \equiv 0$ au plus 3 relations.

$\left.3^{\circ}\right) \quad a(x) \equiv b(x) \equiv e(x) \equiv 0$. Cela fait exactement 3 relations. Je vais montrer ici comment ce dernier cas, où nous avons cessé d'aller plus loin, se rattache lui-même à l'existence de pôles mobiles: je développe l'équation (5) suivant les puissances décroissantes de $y$ : elle est alors $(n$ entier $\geqq 0, \neq 0, \neq-$ I mais pouvant être $\infty$ )

$$
\begin{aligned}
y^{\prime \prime} & =\left(\mathrm{I}-\frac{\mathrm{I}}{n}\right) \frac{y^{\prime 2}}{y}+a_{1}(x) \frac{y^{\prime 2}}{y^{2}}+a_{2}(x) \frac{y^{\prime 2}}{y^{9}}+\cdots \\
& +b_{1}(x) y^{\prime}+b_{2}(x) \frac{y^{\prime}}{y}+b_{3}(x) \frac{y^{\prime}}{y^{2}}+\cdots \\
& +e_{1}(x) y+e_{2}(x)+\frac{e_{3}(x)}{y}+\cdots
\end{aligned}
$$

Si l'on admet l'existence d'un pôle, on a en appelant $i$ l'ordre de ce pôle et en prenant le terme de degré le plus élevé dans chaque ligne: à la première deux termes de degré polaire $i+2$, à la seconde un de degré $i+\mathrm{r}$, ̀̀ la $3^{\text {èmø }}$ un de degré $i$ : les deux termes de degré $i+z$ se détruiront donc, d'où la relation $i=-n$; donc le pôle ne peut exister que si $n$ est fini et négatif: je fais $y=z^{n}$ et vérifie que $z$ vérifie une équation du second ordre pour laquelle $z=0$ est une valeur ordinaire; donc pour $x=x_{0}$ il existe une intégrale $z$ pour laquelle $z_{0}=0$ 
et $z_{0}^{\prime}$ est arbitraire, autrement dit l'équation (5) admet l'intégrale $y=\frac{\lambda}{\left(x-x_{0}\right)^{-n}}+\cdots$ où $\lambda$ est arbitraire.

En répétant cette étude pour $y=0$, I ou $x$, s'il y a lieu, on voit donc qu'on obtient un nombre fini de relations différentielles algébriques entre les coefficients de l'équation (5), par un procédé régulier de calcul.

8. - Le résultat très remarquable obtenu pour les équations du second ordre et du premier degré, rationnelles en $y$, est que l'ensemble des conditions nécessaires obtenues par ce procédé est en même temps suffisant pour que l'intégrale soit à points critiques fixes.

Dans le cas où l'équation contient $y$ non plus rationnellement, mais algébriquement, sans être susceptible d'être ramenée à une autre où $y$ figure rationnellement, c'est-à-dire où la relation $H(y, u)=0$ dont il a été question plus haut est de genre I, il n'en est plus de même, conme M. Paintevé l'avait montré pour l'équation

$$
y^{\prime \prime}=y^{\prime 2}\left\{\frac{y\left[2 k^{2} y-\left(\mathrm{I}+k^{2}\right)\right]}{\left(\mathrm{I}-y^{2}\right)\left(\mathrm{I}-k^{2} y^{2}\right)}+\frac{\mathrm{I}}{\hat{\lambda} \sqrt{\left(\mathrm{I}-y^{2}\right)\left(\mathrm{I}-k^{2} y^{2}\right)}}\right\}
$$

sur cet exemple, l'intégration seule montre qu'on doit écrirc une relation cette fois transcendante et non plus algébrique, que la méthode précédente ne peut donner.

9. Formation explicite de toutes les équations (I) ì points critiques fixes.

Les équations indiquées par M. PaInLevé étaient explicitement connues, par exemple $y^{\prime \prime}=6 y^{2}, y^{\prime \prime}=6 y^{2}-\frac{x}{24}$ ou $y^{\prime \prime}=6 y^{2}+x$, sauf une seule équation

$$
y^{\prime \prime}=-y y^{\prime}+y^{3}-\mathrm{I} 2 q(x) y+\mathrm{I} 2 q^{\prime}(x)
$$

où $q(x)$ vérifie l'équation $q^{\prime \prime}=6 q^{2}+x$ qui est l'une de celles précédemment citées. $\mathrm{Si}$ done on regarde comme connues les transcendantes engendrées par les équations algébriques du $2^{\text {ème }}$ ordre et $\mathrm{du} \mathrm{I}^{\mathrm{er}}$ degré à points critiques fixes, cette équation (E) est donnée explicitement.

Mais dans les cas nouveaux que j'ai mis en évidence je rencontrais des systèmes de conditions différentielles dont l'intégration était, quoiqu'au fond bien simple, assez difficile à apercevoir. Prenons par exemple l'équation 


$$
\begin{aligned}
y^{\prime \prime}=\frac{2}{3}\left(\frac{\mathrm{I}}{y}+\frac{\mathrm{I}}{y-\mathrm{I}}\right) y^{\prime 2}+(a y+ & \left.\frac{b}{y}-\frac{c}{y-\mathrm{I}}+d\right) y^{\prime}+ \\
& +y(y-\mathrm{I})\left[3 a^{2} y+\frac{3 b^{2}}{y^{2}}-\frac{3 c^{2}}{(y-\mathrm{I})^{2}}+h+\frac{k}{y}+\frac{l}{y-\mathrm{I}}\right] .
\end{aligned}
$$

Sur cette forme d'équation où $a, b, \ldots l$ désignent 7 fonctions analytiques de $x$ on a déjà exprimé toutes les conditions que l'on peut obtenir par les équatjons simplifiées. Il y a ensuite à étudier les valeurs singulières $\infty, 0$, I de $y$ : il y a deux familles de pôles mobiles donnant chacune une relation, de même deux familles de zéros et d'unités mobiles, donnant chacune une relation; on obtient ainsi le système

(II) $\left\{\begin{array}{l}h+3\left(a d+a^{2}-a^{\prime}\right)=0 \\ k+3\left(b d+b c+b^{2}-b^{\prime}\right)=0 \\ l+3\left(c a+c b+c d+c^{2}-c^{\prime}\right)=0 \\ \left(2 h+3 a^{2}\right)^{2}+6\left(a h^{\prime}-2 h a^{\prime}\right)-9 a^{3}(b-c)+6 a^{2}(k+l)=0 \\ \left(2 k+3 b^{2}\right)^{2}+6\left(b k^{\prime}-2 k b^{\prime}\right)-9 b^{3}(a+c)+6 b^{2}\left(h-l-3 c^{2}\right)=0 \\ \left(2 l+3 c^{2}\right)^{2}+6\left(c l^{\prime}-2 l c^{\prime}\right)-9 c^{3}(b-a)+6 c^{2}\left(-3 a^{2}-h-k-3 b^{2}\right)=0 .\end{array}\right.$

On verra plus loin comment ces 6 équations permettent d'exprimer les 7 fonctions inconnues au moyen d'une fonction auxiliaire arbitraire et de plus cet exemple montre d'une façon frappante comment, par un mécanisme qui est général, mais qui il était difficile de prévoir, la résolution de ce premier problème, intégration des conditions telles que (II) est intimement liée à l'intégration de l'équation différentielle ( $\mathrm{I}$ ) elle même quand cette intégration est possible ou à la réduction de l'équation à un des 6 types irréductibles I à VI.

D'une manière précise, les relations nécessaires [(II) ou analogues] se laissent intégrer explicitement, en ne considérant comme connus que les éléments analytiques suivants: fonctions rationnelles, exponentielles ou elliptiques, intégrales des 6 équations irréductibles que j'ai données plus haut.

Ces relations nécessaires sont d'ailleurs suffisantes: toute équation du second ordre répondant à ce conditions se ramène soit à une équation linéaire (d'ordre 2,3 ou 4) soit à une équation intégrable (par quadratures, ou par les fonctions elliptiques et dégénérescences), soit à l'un des 6 types irréductibles I à VI.

Pour les équations intégrables, la fixité des points critiques résulte de l'intégration même, pour les autres elle résulte de ce fait que les 6 équations irréductibles I à VI ont, comme nous l'avons dit, leurs points critiques fixes.

Je citerai, parmi celles dont l'intégration etait le plus cachée les équations 


$$
y^{\prime \prime}=\left(x-\frac{I}{n}\right) \frac{y^{\prime 2}}{y}+\left(a y+b+\frac{c}{y}\right) y^{\prime}-\frac{n a^{2}}{(n+2)^{2}} y^{3}+e y^{2}+f y+g+\frac{h}{y}
$$

pour lesquelles j'ai d'ailleurs donné deux méthodes d'intégration.

10. - Je vais maintenant donner plusieurs tableaux de types canoniques, différents suivant le but que l'on se propose.

Je donne d'abord un tableau $T$ d'équations, $Y^{\prime \prime}=F\left(Y^{\prime}, Y, X\right)$ ou $F$ est rationnel en $Y$ et $Y^{\prime}$, qui permet d'obtenir toutes les équations $y^{\prime \prime}=f\left(y^{\prime}, y, x\right)$ où $f$ est rationnel en $y$ et $y^{\prime}$, dont l'intégrale générale est à points critiques fixes. ${ }^{1}$

$S i$ dans le tableau $T$ qui va suivre j'effectue la transformation homographique la plus générale.

$$
Y=\frac{l(x) y+m(x)}{p(x) y+q(x)}, \quad X=r(x)
$$

oit $l, m, p, q, \varphi$ sont des fonctions analytiques quelconques de $x$ jobtiens toutes les équations $d u$ second ordre et du premier degré en $y^{\prime}$, rationnelles en $y$ dont l'intégrale générale est à points critiques fixes.

Ces équations du tableau $T$ sont de la forme

$$
Y^{\prime \prime}=A(Y, X) Y^{\prime 2}+B(Y, X) Y^{\prime}+C(Y, X),
$$

où $A$ est l'une des 8 expressions $\theta^{\prime}$.

Dans ce qui suit $\alpha, \beta, \gamma, \delta, \varepsilon$ sont des constantes numériques; $q(X), r(X), \ldots$ des fonctions analytiques de $X$.

$A \equiv 0$

(I) $Y^{\prime \prime}=0$

(2) $Y^{\prime \prime}=6 Y^{2}$

(3) $Y^{\prime \prime}=6 Y^{2}-\frac{I}{24}$

(4) $Y^{\prime \prime}=6 Y^{\mathbf{2}}+X$

(5) $Y^{\prime \prime}=-3 Y Y^{\prime}-Y^{3}+q(X)\left(Y^{\prime}+Y^{2}\right)$

(6) $Y^{\prime \prime}=-2 Y Y^{\prime}+q(X) Y+q^{\prime}(X) Y$

(7) $Y^{\prime \prime}=2 Y^{3}$

(8) $Y^{\prime \prime}=2 Y^{3}+\alpha Y+\beta$

1 Je rappolle que le cas où $f$ est rationnel en $y$ a seul besoin d'ètre complété. C'eut le seul dont jo m'oceupe dans tout ce mémoire. 
(9) $Y^{\prime \prime}=2 Y^{3}+X Y+a$

(10) $Y^{\prime}=-Y Y^{\prime}+Y^{3}-$ I2 $q(X) Y+$ I2 $q^{\prime}(X)$ avec $q_{X^{2}}^{\prime \prime}=6 q^{2}+S, S=0, \frac{-\mathrm{I}}{24}$ ou $X$. $A \equiv \frac{\mathrm{I}}{\mathrm{Y}}^{\mathrm{l}}$

(II) $\quad Y^{\prime \prime}=\frac{Y^{\prime 2}}{Y}$

(I2) $Y^{\prime \prime}=\frac{Y^{\prime 2}}{Y}+\alpha Y^{3}+\beta Y^{2}+\gamma+\frac{\delta}{Y}$

(13) $\quad Y^{\prime \prime}=\frac{Y^{\prime 2}}{Y}-\frac{Y^{\prime}}{X}+\frac{\mathrm{I}}{X}\left(\alpha Y^{2}+\beta\right)+\gamma Y^{3}+\frac{\delta}{Y}$

(14) $Y^{\prime \prime}=\frac{Y^{\prime 2}}{Y}+q \frac{Y^{\prime}}{Y}-q^{\prime}+r Y Y^{\prime}+r^{\prime} Y^{2}$

(I5) $Y^{\prime \prime}=\frac{Y^{\prime 2}}{Y}+\frac{Y^{\prime}}{Y}+r Y^{2}-Y \frac{d}{d X} \frac{r^{\prime}}{r}$

(16) $Y^{\prime \prime}=\frac{Y^{\prime g}}{Y}-q^{\prime} Y Y^{\prime}-q^{\prime \prime} Y^{2}+q-\frac{\mathrm{I}}{Y}$

$A \equiv\left(\mathrm{I}-\frac{\mathrm{I}}{n}\right) \frac{\mathrm{I}}{Y}, n$ entier $>\mathrm{I}$

(I7) $Y^{\prime \prime}=\left(\mathrm{I}-\frac{\mathrm{I}}{n}\right) \frac{Y^{\prime 8}}{Y}$

(I8) $Y^{\prime \prime}=\frac{Y^{\prime 8}}{2 Y}+4 Y^{2}$

(19) $Y^{\prime \prime}=\frac{Y^{\prime 2}}{2 Y}+4 Y^{9}+2 Y$

(20) $Y^{\prime \prime}=\frac{Y^{\prime 2}}{2 Y}+4 Y^{2}+2 X Y$

(2I) $\quad Y^{\prime \prime}=\frac{3 Y^{\prime 2}}{4}+3 Y^{2}$

(22) $\quad Y^{\prime \prime}=\frac{3 Y^{\prime 2}}{4 Y}-I$

(23) $\quad Y^{\prime \prime}=\frac{3 Y^{\prime 2}}{4 Y}+3 Y^{2}+\alpha Y+\beta$

${ }^{1}$ Ces 2 tableaux pour $A=0$ et $A=\frac{1}{y}$ étaient complets chez M. Pannlevé; je les reproduis ici pour la clarté de ce qui suit. Les modifications que j'ai apportées à ces 2 tableaux m'ont été suggérées aussi pour la simplification des équations que j'ajoute dans les autres tableaux. 
(24) $\quad Y^{\prime \prime}=\left(\mathrm{I}-\frac{\mathrm{I}}{n}\right) \frac{Y^{\prime 2}}{Y}+q Y Y^{\prime}-\frac{n q^{2}}{(n+2)^{2}} Y^{3}+\frac{n q^{\prime}}{n+2} Y^{3}$

(25) $Y^{\prime \prime}=\frac{3}{4} \frac{Y^{\prime 2}}{Y}-\frac{3}{2} Y Y^{\prime}-\frac{Y^{3}}{4}+\frac{q^{\prime}}{2 q}\left(Y^{\prime}+Y^{2}\right)+r Y+q$

(26) $Y^{\prime \prime}=\frac{3}{4} \frac{Y^{\prime 2}}{Y}+6 q^{\prime} \frac{Y^{\prime}}{Y}+3 Y^{2}+12 q Y-12 q^{\prime \prime}-\frac{36 q^{\prime \prime}}{Y}, q^{\prime \prime}=6 q^{2}+S, S=0, \frac{-\mathrm{x}}{24}$ ou $X$

(27) $\quad Y^{\prime \prime}=\left(\mathrm{I}-\frac{\mathrm{I}}{n}\right) \frac{Y^{\prime 2}}{Y}+f_{n}(q, r) Y Y^{\prime}+f_{n}(q, r) Y^{\prime}-\frac{n-2}{n} \frac{Y^{\prime}}{Y}-\frac{n f_{n}^{2}}{(n+2)^{2}} Y^{3}$

$$
+\frac{n\left[f_{n}^{\prime}-f_{n} \varphi_{n}\right]}{n+2} Y^{2}+\psi_{n}(q, r) Y-\varphi_{n}-\frac{\mathrm{I}}{n Y}
$$

$\left(f_{n}, q_{n}, \psi_{n}\right.$ étant 3 fonctions rationnelles de 2 fonctions arbitraires $q, r$ et de leurs dérivées dont le calcul sera indiqué dans le texte, page 54)

(28) $\quad Y^{\prime \prime}=\frac{Y^{\prime 2}}{2 \bar{Y}}-Y Y^{\prime}+q Y^{\prime}+\frac{Y^{3}}{2}-2 q Y^{2}+3\left(q^{\prime}+\frac{q^{2}}{2}\right) Y-\frac{72 r^{2}}{Y}$

(avec $r=\frac{V_{2}-V_{1}}{2}, q=\frac{V_{2}^{\prime}-V^{\prime}}{V_{2}-V_{2}}$ et $V_{1}$ et $V_{2}$ étant deux intégrales quelconques

de $V^{\prime \prime}=6 V^{2}+S, S=0, \frac{-\mathrm{r}}{24}$ ou $\left.X\right)$

(29) $\quad Y^{\prime \prime}=\frac{Y^{\prime 2}}{2} \bar{Y}+\frac{3 Y^{3}}{2}$

(30) $\quad Y^{\prime \prime}=\frac{Y^{\prime 2}}{2 Y}+\frac{3 Y^{3}}{2}+4 \alpha Y^{2}+2 \beta Y-\frac{\gamma^{2}}{2 Y}$

(3I) $\quad Y^{\prime \prime}=\frac{Y^{\prime 2}}{2 \bar{Y}}+\frac{3 Y^{3}}{2}+4 X Y^{2}+2\left(X^{2}-\alpha\right) Y-\frac{\beta^{2}}{2 Y}$

(32) $\quad Y^{\prime \prime}=\frac{Y^{\prime 2}-\mathrm{r}}{2}$

(33) $Y^{\prime \prime}=\frac{Y^{\prime 2}-\mathrm{I}}{2}+4 Y^{2}+c Y$

(34) $\quad Y^{\prime \prime}=\frac{Y^{\prime 2}-I}{2 Y}+4 \alpha Y^{2}-X Y$

(35) $Y^{\prime \prime}=\frac{2}{3} \frac{Y^{\prime 2}}{Y}-\frac{2}{3} Y Y^{\prime}+\frac{2 q}{3} Y^{\prime}+r \frac{Y^{\prime}}{Y}+\frac{2}{3} Y^{3}-\frac{10 q}{3} Y^{2}+\left(4 q^{\prime}+r+\frac{8 q^{2}}{3}\right) Y$

$$
+3\left[\frac{2 q r}{3}-r^{\prime}\right]-\frac{3 r^{2}}{\bar{Y}}
$$


où l'on a $q^{\prime \prime}=2 q^{3}+S q+T, r=-\frac{S}{3}-\frac{2}{3}\left(q^{\prime}+q^{2}\right)$ et $2 q^{3}+S q+T$ désigne l'une quelconque des 3 expressions $2 q^{3}, 2 q^{3}+a q+\beta, 2 q^{3}+X q+a$

$$
\begin{gathered}
Y^{\prime \prime}=\frac{4}{5} \frac{Y^{\prime 2}}{Y}-\frac{2}{3} Y Y^{\prime}-\frac{q}{5} Y^{\prime \prime}+r \frac{Y^{\prime}}{Y}+\frac{4}{5} Y^{3}+\frac{I 4 q}{5} Y^{2}+\left(r-3 q^{\prime}+\frac{6 q^{2}}{5}\right) Y \\
\text { avec } q=\frac{V_{2}^{\prime}-V_{1}^{\prime}}{V_{2}-V_{1}}, \quad r-\frac{q r}{5}-\frac{5 r^{\prime}}{3}-\frac{5 r^{2}}{9} V_{1}+\frac{36}{5} V_{2}-\frac{q}{5}\left[\frac{V_{2}^{\prime}-V_{1}^{\prime}}{V_{2}-V_{1}}\right]^{2}
\end{gathered}
$$

$V_{1}$ et $V_{2}$ désignant 2 intégrales quelconques de $V^{\prime \prime}=6 V^{2}+S, S=0,-\frac{\mathrm{I}}{24}$ ou $X$.

Si $V_{2}$ tend vers $V_{1}$ (cette remarque s'applique à tous les cas analogues) que fant-il entendre par $\frac{V_{2}^{\prime}-V^{\prime}{ }^{\prime}}{V_{2}-V_{1}}$ ? Soit $V(X, \alpha, \beta)$ lintégrale générale de $V^{\prime \prime}=6 V^{2}+X$ par exemple; $V_{1}$ est obtenu en donnant à $\alpha$ et $\beta$ les valeurs numériques $\alpha_{1}$ et $\beta_{1}$; soit de même $\frac{\partial V}{\partial a}(X, \alpha, \beta)$ j'appellerai $\frac{\partial V}{\partial a}(X, \alpha, \beta)$ cette dérivée où j'ai remplacé $a$ et $\beta$ par $\alpha_{1}$ et $\beta_{1}$ : dans ces conditions si $V_{2}$ tend vers $V_{1}$ on considérera $\frac{V_{2}^{\prime}-V_{1}^{\prime}}{V_{2}-V_{1}}$ comme représentant $\frac{\lambda \frac{\partial^{2} V_{1}}{\partial \alpha \partial X}+\mu \frac{\partial^{2} V_{1}}{\partial \beta \partial X}}{\partial \frac{\partial V_{1}}{\partial \alpha}+\mu \frac{\partial V_{1}}{\partial \beta}}$ où $i$ et $"$ sont deux constantes numériques arbitraires.

$A=\frac{\mathrm{I}}{2 Y}+\frac{\mathrm{I}}{Y-\mathrm{I}}$

(37) $Y^{\prime \prime}=Y^{\prime 2}\left(\frac{I}{2 Y}+\frac{I}{Y-I}\right)$

(38) $Y^{\prime \prime}=Y^{\prime 2}\left(\frac{\mathrm{I}}{2 Y}+\frac{\mathrm{I}}{Y-I}\right)+Y(Y-\mathrm{I})\left[\alpha(Y-\mathrm{I})+\beta \frac{(Y-\mathrm{I})}{\bar{Y}^{\bar{y}}}+\frac{y}{Y-\mathrm{I}}+\frac{\delta}{(Y-I)^{2}}\right]$

(39) $Y^{\prime \prime}=Y^{\prime 2}\left(\frac{\mathrm{I}}{2 Y}+\frac{\mathrm{I}}{Y-\mathrm{I}}\right)-\frac{Y^{\prime}}{X}+\frac{(Y-\mathrm{I})^{2}}{X^{2}}\left(a Y+\frac{\beta}{Y}\right)+\gamma^{\prime} \bar{X}+\frac{\delta Y(Y+\mathrm{I})}{Y-\mathrm{I}}$

(40) $Y^{\prime \prime}=Y^{\prime 2}\left(\frac{I}{2 Y}+\frac{I}{Y-I}\right)+\frac{2(q Y+r)}{Y-I} Y^{\prime}+\frac{(Y-I)^{2}}{2}\left(s^{2} Y-\frac{t}{Y}\right)+$ $+2\left[q^{2}-r^{2}-\left(q^{\prime}+r^{\prime}\right)\right] I^{\prime}$

$\operatorname{avec} s^{\prime}-2 q s=0, t^{\prime}+2 t r=0$ 
$A \equiv \frac{2}{3}\left(\frac{\mathrm{I}}{Y}+\frac{\mathrm{I}}{Y-\mathrm{I}}\right)$

(4I) $\quad Y^{\prime \prime}=\frac{2}{3}\left(\frac{I}{Y}+\frac{I}{Y-I}\right) Y^{\prime 2}$

(42) $Y^{\prime \prime}=\frac{2}{3}\left(\frac{I}{Y}+\frac{I}{Y-I}\right) Y^{\prime 2}+\left[q Y+\frac{r}{Y}-\frac{s}{Y-I}-\frac{q+r+s}{2}\right] Y^{\prime}$

$$
\begin{array}{r}
+Y(Y-\mathrm{I})\left[3 q^{2} Y+\frac{3 r^{2}}{Y^{2}}-\frac{3 s^{2}}{(Y-\mathrm{I})^{2}}+3 q^{\prime}+\frac{3 q}{2}(r+s-q)+\frac{3 r^{\prime}-\frac{3 r}{2}(r+s-q)}{Y}+\right. \\
\left.+\frac{\left.3 s^{\prime}-\frac{3 s}{2}(q+s+r)\right]}{Y-\mathrm{I}}\right]
\end{array}
$$

avec

$$
\begin{aligned}
& 3 q=\frac{V^{\prime}}{V}-V+\frac{E}{V}-2 C \\
& 3 r=\frac{V^{\prime}}{V}+V+\frac{E}{V}+2 C \\
& 3 s=2 V \\
& V^{\prime \prime}=\frac{V^{\prime 2}}{2 V}+\frac{3}{2} V^{3}+4 C V^{2}+2 D V-\frac{E^{2}}{2 V}
\end{aligned}
$$

cette dernière équation représentant l'une quelconque des équations rencontrées précédemment

$$
\begin{aligned}
& V^{\prime \prime}=\frac{V^{\prime 2}}{2 V}+\frac{3}{2} V^{3} \\
& V^{\prime \prime}=\frac{V^{\prime 2}}{2 V}+\frac{3}{2} V^{3}+4 \alpha V^{2}+2 \beta V-\frac{\gamma^{2}}{2} \bar{V} \\
& V^{\prime \prime}=\frac{V^{\prime 2}}{2 V}+\frac{3}{2} V^{3}+4 X V^{2}+2\left(X^{2}-\alpha\right) V-\frac{\beta^{2}}{2 V} .
\end{aligned}
$$

$A \equiv \frac{3}{4}\left(\frac{I}{Y}+\frac{\mathrm{I}}{Y-\mathrm{I}}\right) Y^{\prime 2}$

(43) $\quad Y^{\prime \prime}=\frac{3}{4}\left(\frac{\mathrm{I}}{Y}+\frac{\mathrm{I}}{Y-I}\right) Y^{\prime 2}$

(44) $Y^{\prime \prime}=\frac{3}{4}\left(\frac{\mathrm{I}}{Y}+\frac{\mathrm{I}}{Y-\mathrm{I}}\right) Y^{\prime 2}+Y(Y-\mathrm{I})\left[\frac{\alpha}{Y}+\frac{\beta}{Y-\mathrm{I}}+2 \gamma\left(2 Y^{\prime}-\mathrm{I}\right)\right]$ 
Équations différentielles dont l'integrale générale est à points critiques fixes.

(45) $\quad Y^{\prime \prime}=\frac{3}{4}\left(\frac{I}{Y}+\frac{I}{Y-I}\right) Y^{\prime 2}+\left(a+\frac{b}{Y}-\frac{c}{Y-I}\right) Y^{\prime}+$

$$
+Y(Y-\mathrm{I})\left[8 D^{2}\left(Y-\frac{\mathrm{I}}{2}\right)+\frac{b^{2}}{Y^{2}}-\frac{c^{2}}{(Y-\mathrm{I})^{2}}+\frac{h}{Y}+\frac{k}{Y-\mathrm{I}}\right]
$$

avec $D=\frac{V_{2}-V_{1}}{2}, \quad b-c=-\frac{3}{2}\left(V_{1}+V_{2}\right), a=\frac{V_{2}^{\prime}-V_{1}^{\prime}}{V_{2}-V_{1}}$,

$$
\begin{gathered}
b+c=-\frac{3}{2} \frac{V_{2}^{\prime}-V_{1}^{\prime}}{V_{2}-V_{1}}, \\
h=2 b^{\prime}+a b, \quad k=2 c^{\prime}+a c
\end{gathered}
$$

et $V_{1}, V_{2}$ étant deux intégrales quelconques de l'une des 3 équations $V^{\prime \prime}=2 V^{3}$ ou $V^{\prime \prime}=2 V^{3}+\alpha V+\beta, V^{\prime \prime}=2 V^{3}+X V+\alpha$.

$\left(4^{6)}\right.$

$$
\begin{gathered}
Y^{\prime \prime}=\frac{3}{4}\left(\frac{\mathrm{I}}{Y}+\frac{\mathrm{I}}{Y-\mathrm{I}}\right) Y^{\prime 2}-\left[\frac{h^{\prime}}{h}+\frac{3}{2} \frac{h^{\prime}}{\mathrm{h}-\mathrm{I}}\right] Y^{\prime}+ \\
+\left[\frac{8 \beta^{2}}{h^{2}}\left(Y-\frac{\mathrm{I}}{2}\right)-\frac{9}{4}\left(\begin{array}{l}
h^{\prime} \\
\bar{h}^{2}
\end{array}\right)^{2} \frac{\mathrm{I}}{(Y-\mathrm{I})^{2}}+\frac{h}{Y}+\left(\frac{3 h^{\prime \prime}}{h}-\frac{9 h^{\prime 2}}{2 h^{2}}\right) \frac{\mathrm{I}}{Y-\mathrm{I}}\right] Y(Y-\mathrm{I}) \\
\text { avec } q^{\prime \prime}=2 q^{3}+a q+\beta, h=2\left(q^{\prime}+q^{2}\right)+\alpha
\end{gathered}
$$

(47) $Y^{\prime \prime}=\frac{3}{4}\left(\frac{\mathrm{I}}{Y}+\frac{\mathrm{I}}{Y-\mathrm{I}}\right) Y^{\prime 2}-\frac{h^{\prime}}{h}\left[\mathrm{I}+\frac{3}{2} \frac{\mathrm{I}}{Y-\mathrm{I}}\right] Y^{\prime \prime}$

$$
\begin{gathered}
+\left[\frac{8\left(\alpha+\frac{\mathrm{I}}{2}\right)^{2}}{h^{2}}\left(Y-\frac{\mathrm{I}}{2}\right)-\frac{9}{4}\left(\frac{h^{\prime}}{h}\right)^{2} \frac{\mathrm{I}}{(Y-\mathrm{I})^{2}}+\frac{h}{Y}+\left(\frac{3 h^{\prime \prime}}{h}-\frac{9 h^{\prime 2}}{2 h^{2}}\right) \frac{\mathrm{I}}{Y-\mathrm{I}}\right] Y(Y-\mathrm{I}) \\
\text { avec } q^{\prime \prime}=2 q^{3}+X q+\alpha, \quad h=2\left(q^{\prime}+q^{2}\right)+X .
\end{gathered}
$$

$A=\frac{\mathrm{I}}{2}\left(\frac{\mathrm{I}}{Y}+\frac{\mathrm{I}}{Y-\mathrm{I}}+\frac{\mathrm{I}}{Y-\alpha}\right)$

(48) $\quad Y^{\prime \prime}=\frac{Y^{\prime 2}}{2}\left(\frac{I}{Y}+\frac{I}{Y-I}+\frac{I}{Y-\alpha}\right)+$

$$
+Y(Y-\mathrm{I})(Y-\alpha)\left[\frac{\beta}{Y^{z}}+\frac{\gamma}{(Y-\mathrm{I})^{z}}+\frac{\delta}{(Y-\alpha)^{2}}+\varepsilon\right]
$$

$A=\frac{\mathrm{I}}{2}\left(\frac{\mathrm{I}}{\bar{Y}}+\frac{\mathrm{I}}{Y-\mathrm{I}}+\frac{\mathrm{I}}{Y-X}\right)$

(49)

$$
\begin{aligned}
Y^{\prime \prime}=\frac{Y^{\prime 2}}{2}\left(\frac{\mathrm{I}}{Y}+\right. & \left.\frac{\mathrm{I}}{Y-\mathrm{I}}+\frac{\mathrm{I}}{Y-X}\right)-Y^{\prime}\left[\frac{\mathrm{I}}{X}+\frac{\mathrm{I}}{X-\mathrm{I}}+\frac{\mathrm{I}}{Y-X}\right] \\
& +\frac{Y(Y-\mathrm{I})(Y-X)}{2 X^{2}(X-\mathrm{I})^{2}}\left[\alpha-\frac{\beta X}{Y^{2}}+\frac{\gamma(X-\mathrm{I})}{(Y-\mathrm{I})^{2}}-\frac{(\delta-\mathrm{I}) X(X-\mathrm{I})}{(Y-X)^{2}}\right]
\end{aligned}
$$




$$
\begin{aligned}
& A \equiv \frac{2}{3 Y}+\frac{\mathrm{I}}{2(Y-\mathrm{I})} \\
& \text { (50) } \quad Y^{\prime \prime}=Y^{\prime 2}\left(\frac{2}{3 \bar{Y}}+\frac{I}{2(Y-I)}\right)+\left(a Y+b+\frac{c}{Y}\right) Y^{\prime}+ \\
& +\left[\frac{3 a^{2}}{8} Y+f+\frac{3 c^{2}}{Y^{2}}+\frac{h}{(Y-\mathrm{I})^{2}}+\frac{k}{Y}+\frac{h}{3(Y-\mathrm{I})}\right] Y(Y-\mathrm{I}) \\
& \text { avec } a=-\frac{\text { Io }}{9}(t+u), c=-\frac{4}{9}(u-2 t), b=\frac{2 t+5 u}{9}, h=-\frac{9 H^{2}}{2} \text {, } \\
& 2 k=6\left(c^{\prime}-b c\right)-3 c^{2}, \quad f=\frac{3}{2}\left(a^{\prime}-a b\right)-\frac{3 a^{2}}{4}, \\
& t=\left[\frac{V_{2}^{\prime}-V_{1}^{\prime}}{V_{2}-V_{1}}+\frac{V_{3}^{\prime}-V_{1}^{\prime}}{V_{3}-V_{1}}\right], \quad H=\left[\frac{V_{2}^{\prime}-V_{1}^{\prime}}{V_{2}-V_{1}}-\frac{V^{\prime}{ }_{3}-V_{1}^{\prime}}{V_{3}-V_{1}}\right], \quad u=-\frac{H^{\prime}}{H}
\end{aligned}
$$

$V_{1}, \quad V_{2}, V_{3}$ étant 3 intégrales particulières de $V^{\prime \prime}=6 V^{2}+S, S=0, \frac{-\mathrm{I}}{24}$, ou $X .{ }^{1}$

11. - Problème inverse.

Etant donnée une équation $y^{\prime \prime}=P\left(y^{\prime}, y, x\right)$ oì $P$ est rationnel en $y^{\prime}$ et $y$ comment reconnaître si elle a ses points critiques fixes?

Il suffit de vérifier si une transformation

$$
Y=\frac{l(x) y+m(x)}{p(x) y+\frac{m}{q(x)}, \quad X=r(x)}
$$

permet de ramener l'équation à l'une des formes donnée dans le tableau $T$. Mais comment se calculent les coefficients $l, m, p, q, q$ de cette transformation?

Pour les transcendantes irréductibles, $l, m, p, q, q$ sont des combinaisons algébriques de l'équation proposée. Mais pour les autres il n'en est plus de même, au moins en général; $l, m, p, q$ s'expriment algébriquement au moyen de $\varphi$ et $\varphi^{\prime}$; quant à la fonction $\varphi$ elle est donnée soit par une ou deux quadratures soit par l'intégration d'une équation différentielle du second ordre. Les opérations qu'exige le calcul de cette transformation sont d'ailleurs les mêmes que celles qu'exige l'intégration de l'équation elle même: intégration qui se ramène,

1 Si $V_{2}$ on $V_{3}$ tendent vers $V_{1}$, ou si $V_{2}$ et $V_{8}$ tendent simultanément vers $V_{1}$ ou si $V_{3}$ tend vers $V_{2}$, on aura à trouver pour $t, H$ ou $u$ les valeurs limites de quotients de fonctions prenant la forme $\frac{o}{0}$. Cette limite sera indiquée dans le texte et se fait comme il a été indiqué précédemment, page 15. On voit que pour l'équation (5o) il y aura un nombre considérade types particuliers. 
Équations différentielles dont l'intégrale générale est à points critiques fixes.

nous l'avons vu, soit à des quadratures soit à l'intégration d'une équation linéaire d'ordre 4 au plus.

Il est alors plus avantageux, pour traiter le problème inverse, de dresser un nouveau tableau $\Theta$ des types canoniques auxquels les équations $y^{\prime \prime}=P\left(y^{\prime}, y, x\right)$ se laissent ramener algébriquement par une transformation

$$
\eta=\frac{\lambda(x) y+\mu(x)}{x(x) y+\chi(x)}, \quad \xi=\varphi(x)
$$

$\lambda, \iota, \pi, \chi, \varphi$ se calculant cette fois algébriquement. Ce tableau $\Theta$ est trop considérable pour pouroir le donner explicitement: il faudrait reprendre chacune des 50 types du tableau $T$ et lui faire correspondre dans le tableau $\Theta$ un certain nombre d'équations en nombre considérable: la dernière équation (50) par exemple conduit à plus de roo types. - Les équations (28), (35), (36), (42), (45), (46), (47) donnent aussi un nombre considérable d'équations correspondantes dans le tableau $\Theta$.

Je me bornerai done à prendre comme exemple les équations (29), (30), (3I). Le raisonnement étant lo même pour toutes, le genre de calcul le même, il suffira de quelques indications rapides pour permettre de reconstituer les types $\Theta$ dans tous les cas.

12. - Je vais maintenant extraire du tableau $\mathrm{T}$ un tableau $(t)$, dont il suffit d'indiquer la nature de chaque équation pour en déduire immédiatement la nature de toutes les équations $T$ et en donner l'intégration effective, quand on regarde comme complètement connus les éléments analytiques du tableau $(t)$. C'est le tableau suivant'

$$
\begin{aligned}
& \text { (1) } Y^{\prime \prime}=0 \\
& \text { (2) } Y^{\prime \prime}=6 Y^{2} \\
& \text { (3) } Y^{\prime \prime}=6 Y^{2}-\frac{1}{24} \\
& \text { (4) } Y^{\prime \prime}=6 Y^{2}+X \\
& \text { (5) } Y^{\prime \prime}=-3 Y Y^{\prime}-Y^{3}+q(X)\left(Y^{\prime}+Y^{2}\right) \\
& \text { (6) } Y^{\prime \prime}=-2 Y Y^{\prime}+q(X) Y^{\prime}+q^{\prime}(X) Y \\
& \text { (7) } Y^{\prime \prime}=2 Y^{3} \\
& \text { (8) } Y^{\prime}=2 Y^{3}+\alpha Y+\beta
\end{aligned}
$$

\footnotetext{
${ }^{1}$ Voir pour chaque équation $(t)$ ce qui en a été dit au tableau $T$.
} 

(9) $Y^{\prime \prime}=2 Y^{3}+X Y+\alpha$
(10) $Y^{\prime \prime}=\frac{Y^{\prime 2}}{Y}$
(II) $\quad Y^{\prime \prime}=\frac{Y^{\prime 2}}{Y}+\alpha Y^{3}+\beta Y^{2}+\gamma+\frac{\delta}{Y}$
(12) $\quad Y^{\prime \prime}=\frac{Y^{\prime 2}}{Y}-\frac{Y^{\prime}}{X}+\frac{1}{X}\left(\alpha Y^{2}+\beta\right)+\left(\gamma Y^{3}+\frac{\delta}{Y}\right)$
(13) $Y^{\prime \prime}=\frac{Y^{\prime 8}}{Y}+q \frac{Y^{\prime}}{Y}-q^{\prime}+r Y Y^{\prime}+r^{\prime} Y^{2}$
(I4) $Y^{\prime \prime}=\left(\mathrm{I}-\frac{\mathrm{I}}{n}\right) \frac{Y^{\prime 2}}{Y}+q^{\prime} Y Y-\frac{n q^{2}}{(n+2)^{2}} Y^{3}+\frac{n q^{\prime}}{n+2} Y^{\prime}$
(I5) $\quad Y^{\prime \prime}=\left(\mathrm{I}-\frac{\mathrm{I}}{n}\right) \frac{Y^{\prime 8}}{Y}+f_{n}(q, r) Y Y^{\prime}+\varphi_{n}(q, r) Y^{\prime}-\frac{n-2}{n} \frac{Y^{\prime}}{Y}-\frac{n f_{n}^{2}}{(n+2)^{2}} Y^{3}$ $+\frac{n\left(f_{n}^{\prime}-f_{n}\left(f_{n}\right)\right.}{n+2} Y^{2}+\psi_{n}(q, r) Y-p_{n}-\frac{\mathrm{I}}{n Y}$

(16) $Y^{\prime \prime}=\frac{Y^{\prime 8}}{2 Y}+\frac{3 Y^{3}}{2}$

(I7) $Y^{\prime \prime}=\frac{Y^{\prime d}}{2 Y}+\frac{3 Y^{3}}{2}+4 \alpha Y^{2}+2 \beta Y-\frac{\gamma^{2}}{2 Y}$

(I8) $Y^{\prime \prime}=\frac{Y^{\prime 2}}{2 Y}+\frac{3 Y^{3}}{2}+4 X Y^{2}+2\left(X^{2}-\alpha\right) Y-\frac{\beta^{2}}{2 Y}$

(I9) $Y^{\prime \prime}=\frac{Y^{\prime 2}-I}{2} \bar{Y}$

(20) $Y^{\prime \prime}=Y^{\prime 2}\left(\frac{\mathrm{I}}{2 Y}+\frac{\mathrm{I}}{Y-\mathrm{I}}\right)$

(2I) $Y^{\prime \prime}=Y^{\prime 2}\left(\frac{\mathrm{I}}{2 Y}+\frac{\mathrm{I}}{Y-\mathrm{I}}\right)+Y(Y-\mathrm{I})\left[\alpha(Y-\mathrm{I})+\beta \frac{(Y-\mathrm{I})}{Y^{g}}+\frac{\gamma}{Y-\mathrm{I}}+\frac{\delta}{(Y-\mathrm{I})^{2}}\right]$

(22) $Y^{\prime \prime}=Y^{\prime 2}\left(\frac{I}{2 Y}+\frac{I}{Y-I}\right)-\frac{Y^{\prime}}{X}+\frac{(Y-I)^{2}}{X^{3}}\left(\alpha Y+\frac{\beta}{Y}\right)+\gamma \frac{Y}{X}+\frac{\delta Y(Y+I)}{Y-I}$

(23) $Y^{\prime \prime}=\frac{Y^{\prime 2}}{2}\left(\frac{I}{Y}+\frac{I}{Y-I}+\frac{I}{Y-\alpha}\right)+$

$$
+Y(Y-1)(Y-\alpha)\left[\beta+\frac{\gamma}{Y^{2}}+\frac{\delta}{(Y-I)^{2}}+\frac{\epsilon}{(Y-\alpha)^{8}}\right]
$$




$$
\text { (24) } \begin{aligned}
Y^{\prime \prime}=\frac{Y^{\prime 2}}{2}\left(\frac{I}{Y}\right. & \left.+\frac{I}{Y-I}+\frac{I}{Y-X}\right)-Y^{\prime}\left[\frac{\mathrm{I}}{X}+\frac{\mathrm{I}}{X-\mathrm{I}}+\frac{\mathrm{I}}{Y-\bar{X}}\right] \\
& +\frac{Y(Y-\mathrm{I})(Y-X)}{2 X^{2}(X-\mathrm{I})^{2}}\left[\alpha-\beta \frac{X}{Y^{2}}+\gamma \frac{(X-\mathrm{I})}{(Y-\mathrm{I})^{2}}-\frac{(\delta-\mathrm{I}) X(X-\mathrm{I})}{(Y-X)^{2}}\right] .
\end{aligned}
$$

Remarquons en passant que les coefficients de toutes les équations $(t)$ sont explicitement connus. Ces coefficients sont rationnels en $X$, sauf pour 5 d'entre elles: (5), (6), ( $\left.\mathrm{I}_{3}\right),\left(\mathrm{I}_{4}\right),\left(\mathrm{I}_{5}\right)$ où figurent des fonctions analytiques $q, r$ arbitraires de $X$ et leurs dérivées jusqu'à un ordre fini. Ces 5 équations sont d'ailleurs intégrables.

13. - Toute équation de $T$ non contenue dans $(t)$ dérive d'une équation du tableau $(t)$ par le procédé suivant.

Pour abréger le langage j'appellerai équation secondaire l'équation $Y^{\prime \prime}=$ $p\left(Y^{\prime}, Y, X\right)$ considérée dans $T$ et $V^{\prime \prime}=P\left(V^{\prime}, V, X\right)$, l'équation de $(t)$ à laquelle elle se ramène, (pour la clarté j'ai remplacé $Y$ par $V$ pour l'équation de $t$ ), sera dite équation primaire.

J'apprends à former pour l'équation secondaire $Y^{\prime \prime}=p\left(Y^{\prime}, Y, X\right)$ une fonction $V=F\left(Y^{\prime}, Y, X\right)$ rationnelle en $Y^{\prime}$ et $Y$ telle qu'inversement j'aie $Y=$ $\varphi\left(V^{\prime}, V, X\right), \varphi$ étant rationnelle en $V^{\prime}$ et $V$. Le système différentiel

$$
\left\{\begin{array}{l}
V=F\left(Y^{\prime}, Y, X\right) \\
Y=\varphi\left(V^{\prime}, V, X\right)
\end{array}\right.
$$

équivaut à l'équation secondaire, qu'on obtient par élimination de $V$, tandis que l'élimination de $Y$ conduit à l'équation primaire.

Or l'équation primaire a ses coefficients explicitement connus; voyons comment on obtient les coefficients des fractions rationnelles $\varphi\left(V^{\prime}, V, X\right), F\left(Y^{\prime}, Y, X\right)$ et $p\left(Y^{\prime}, Y, X\right)$ : Appelons $V(X, a, \beta)$ l'intégrale générale du type canonique primaire et $V_{j}$ l'intégrale particulière correspondant aux valeurs numériques $\alpha_{j}$ et $\beta_{j}$ données à $\alpha$ et $\beta$; soient de même $\frac{\partial V_{j}}{\partial X^{\prime}}, \frac{\partial V_{j}}{\partial \alpha}, \frac{\partial^{2} V_{j}}{\partial \alpha \partial \beta}, \frac{\partial^{2}}{\partial X} \frac{V_{j}}{\partial \alpha}, \ldots$ les dérivées de $V$ où l'on fait $\alpha=\alpha_{j}, \beta=\beta_{j}$; la fonction $\varphi\left(V^{\prime}, V, X\right)$ rationnelle en $V$ et $V^{\prime}$ a pour coefficients des combinaisons rationnelles de 3 (au plus) intégrales particulières $V_{j}$, soient $V_{1}, V_{2}$ et $V_{3}$ et des dérivées $\frac{\partial V_{j}}{\partial X}, \frac{\partial V_{j}}{\partial \alpha}, \cdots \frac{\partial^{2}}{\partial X} \frac{V_{j}}{\partial \alpha}, \cdots$ les dérivées étant au plus du $3^{\text {ème }}$ ordre; or quand on fait sur l'équation primaire $V^{\prime \prime}=$ $P\left(V^{\prime}, V, X\right)$ une telle transformation $Y=\varphi\left(V^{\prime}, V, X\right)$ il est bien clair que l'équation secondaire obtenue $Y^{\prime \prime}=p\left(Y^{\prime}, Y, X\right)$ a ses coefficients nécessairement transcendants et que ces coefficients s'expriment d'une façon toute sembable au moyen 
B. Gambier.

de $V_{1}, V_{2}, V_{3}$. C'est ce que l'on constate immédiatement sur le tableau $T$. Ceci explique aussi ce que j'annonçais au paragraphe 9, à savoir que la détermination des coefficients des équations étudiées [c'est-à-dire intégration de systèmes différentiels tels que II] et que l'intégration de l'équation elle même, si cette intégration est possible, ou la réduction de cette équation à un type irréductible, constituent en réalité le même problème: tant qu'on n'avait pas aperçu cette identité des 2 questions, difficile à priori à apercevoir, les efforts pour résoudre l'une ou l'autre devaient rester stériles.

14. Conclusion. Il est très remarquable que les équations $y^{\prime \prime}=R\left(y^{\prime}, y, x\right)$ où $R$ est rationnel en $y^{\prime}$, algébrique ou rationnel en $y$ dont l'intégrale générale est à points critiques fixes puissent être définies explicitement soit à l'aide de types canoniques algébriques soit à l'aide de types canoniques renfermant la variable $X$ sous forme transcendante mais alors exprimables rationnellement au moyen des transcendantes engendrées par les types canoniques algébriques.

Qu'il me soit permis d'exprimer ici à M. PaInlevé toute la reconnaissance que je lui dois pour les conseils et les encouragements qu'il m'a prodigués au cours de mes recherches.

\section{CHAPITRE I.}

Equations $y^{\prime \prime}=\left(\mathrm{I}-\frac{\mathrm{I}}{n}\right) \frac{y^{\prime 2}}{y}+B(y, x) y^{\prime}+C(y, x)$

1. - $B$ et $C$ doivent être des fractions rationnelles en $y$ n'ayant d'autre pôle que $y=0$, donc $B \equiv \frac{P(y, x)}{y}, C \equiv \frac{Q(y, x)}{y}$ où $P$ et $Q$ sont deux polynômes en $y$. La transformation y $Y=\mathrm{I}$ montre immédiatement que $P$ est du second degré, $Q \mathrm{du} 3^{\text {ème }}$. On peut donc écrire l'équation sous la forme $(a, b, \ldots h$ étant analytiques en $x$ ),

$$
y^{\prime \prime}=\left(\mathrm{I}-\frac{\mathrm{I}}{n}\right) \frac{y^{\prime 2}}{y}+y^{\prime}\left(a y+b+\frac{c}{y}\right)+d y^{3}+e y^{2}+f y+g+\frac{h}{y}
$$

si l'on pose $y Y=\mathrm{I}$ elle devient 


$$
Y^{\prime \prime}=\left(\mathrm{I}+\frac{\mathrm{I}}{n}\right) \frac{Y^{\prime 2}}{Y}+Y^{\prime}\left(c Y+b+\frac{a}{Y}\right)-h Y^{3}-g Y^{2}-f Y-e-\frac{d}{Y^{3}} .
$$

Ceci montre bien qu'il est inutile de considérer les valeurs négatives de l'entier $n$; pourtant dans la discussion il $\mathrm{y}$ a quelquefois avantage à laisser indéterminé le signe de $n$ : ceci permettra par exemple de déduire immédiatement l'étude de la valeur $y=0$ de l'étude de $y=\infty$ : car en appliquant les résultats trouvés pour $y=\infty$ à l'équation (2) on obtient les résultats relatifs à $y=0$ pour l'équation (I). Ce n'est qu'en fin de discussion que l'on se bornera au cas de $n$ positif.

La simplifiée que l'on doit former avant l'étude de $y=\infty$ s'obtient par $x=x_{0}+\alpha X, Y=\alpha y$, substituant dans (I) et annulant ensuite $\alpha$. On a $\left[a_{0}=a\left(x_{v}\right)\right.$, $\left.d_{0}=d\left(x_{0}\right)\right]$.

$$
y^{\prime \prime}=\left(\mathrm{I}-\frac{\mathrm{I}}{n}\right) \frac{y^{\prime 2}}{y}+a_{0} y y^{\prime}+d_{0} y^{3}
$$

Je pose $y^{\prime}=u y^{2}$, comme j'ai dit dans l'introduction, d'où l'équation

$$
\frac{u^{3}}{u^{\prime 2}}=\frac{-\left(\frac{\mathrm{I}}{n}+\mathrm{I}\right) 2 u+a_{0}}{-\left(\frac{\mathrm{I}}{n}+\mathrm{I}\right) u^{2}+a_{0} u+d_{0}}+\frac{u}{-\left(\mathrm{I}+\frac{\mathrm{I}}{n}\right) u^{2}+a_{0} u+d_{0}} .
$$

Il suffit de décomposer le second membre de (4) en éléments simples, ce qui est immédiat pour la $I^{\text {ère }}$ fraction, puis d'exprimer que ce second membre a l'une des formes de BRIOT et BoUQUET: il suffit dans les expressions $\theta$ données plus haut, page 2 de remplacer $y$ par $u$. La discussion est extrêmement simple, en remarquant qu'ici je ne peux avoir que deux fractions au plus et que la somme des résidus est égale à $I+\frac{I}{n+I}$. Si par exemple je veux avoir la fieme forme de $\theta$ je dois la réduire à $\frac{2}{3}\left(\frac{\mathrm{r}}{u-\alpha}+\frac{\mathrm{I}}{u-\beta}\right)$ et alors j'ai immédiatement $n-2$, $a_{0}=0$ c'est-à-dire $n=2, a(x) \equiv 0$ et de même dans les autres cas. Je donne ici le résumé de la discussion.

2. Si $n$ est quelconque on peut avoir l'une ou l'autre des hypothèses suivantes

$$
a(x) \equiv d(x) \equiv \text { o } \quad \text { ou } \quad d(x) \equiv-\frac{n a^{2}(x)}{(n+2)^{2}}
$$

Il existe trois valeurs particulières de $n$ qui, en outre de l'une ou l'autre de ces hypothèses, peuvent donner d'autres hypothèses admissibles: 
Pour $n=2$ on peut avoir ou $a(x) \equiv 0, d \equiv$ o ou $d(x) \equiv \frac{a^{2}}{2}$.

Pour $n=3$ on peut avoir $d=\frac{3 a^{2}}{2}$.

Pour $n=5$ on peut avoir $d \equiv 5 a^{2}$.

D'après la remarque faite plus haut cette discussion sert en même temps à indiquer les relations possibles entre $c$ et $h$ qui sont:

pour $n$ quelconque ou $c \equiv h \equiv$ o ou $h=-\frac{n c^{2}}{(n-2)^{2}}$ et pour $n=2$ ou $c \equiv h \equiv 0$ ou $c \equiv 0, h \equiv \equiv$. On remarquera d'ailleurs qu'ici le cas de $n=2$ ne donne somme tonte rien de distinct, pour $c$ et $h$, du cas de $n$ quelconque.

3. Si l'on a $a \equiv d \equiv 0$, il faut former la seconde simplifiée en substituant $x=x_{0}+\alpha X, Y=\alpha^{2} y$ dans (I):

$$
Y^{\prime \prime}=\left(\mathrm{I}-\frac{\mathrm{I}}{n}\right) \frac{Y^{\prime 2}}{Y}+e_{0} Y^{2}
$$

$e(x) \equiv 0$ est une solution acceptable, sinon on pose $Y^{\prime}=n u Y$ d'où $e_{\| \prime} Y=n\left[u^{\prime}+u^{2}\right]$ : $u^{\prime \prime}=(n-2) u u^{\prime}+n u^{3}$. Cette équation en $u$ donne, d'après les résultats de M.

Painleví les 3 cas: $n=2, n=4, n=-4$, en écartant $n= \pm \mathrm{I}$.

Donc $a \equiv d \equiv$ entraîne $e \equiv 0$ sauf si $n=2$ ou si $n=4$ et de même $c=h \equiv 0$ entraîne $g \equiv 0$ sauf si $n=4$.

4. Les résultats précédents conduisent à écrire I4 types et I4 seulement d'équations à étudier

(I)

$$
\left\{\begin{array}{l}
y^{\prime \prime}=\frac{y^{\prime 2}}{y}\left(\mathrm{x}-\frac{\mathrm{I}}{n}\right)+b y^{\prime}+f y \\
y^{\prime \prime}=\frac{y^{\prime 2}}{2 y}+b y^{\prime}+e y^{2}+f y \\
y^{\prime \prime}=\frac{3 y^{\prime 2}}{4 y}+b y^{\prime}+e y^{2}+f y+g
\end{array}\right.
$$

(2) $\left\{\begin{array}{l}y^{\prime \prime}=\frac{y^{\prime 2}}{y}\left(\mathrm{I}-\frac{\mathrm{I}}{n}\right)+(a y+b) y^{\prime}-\frac{n a^{2}}{(n+2)^{2}} y^{3}+e y^{2}+f y \\ y^{\prime \prime}=\frac{3 y^{\prime 2}}{4 y}+(a y+b) y^{\prime}-\frac{a^{2}}{9} y^{3}+e y^{2}+f y+g\end{array}\right.$

(3) $\left\{\begin{array}{l}y^{\prime \prime}=\frac{y^{\prime 2}}{y}\left(\mathrm{I}-\frac{\mathrm{I}}{n}\right)+\left(b+\frac{c}{y}\right) y^{\prime}+f y+g-\frac{n c^{2}}{(n-2)^{2}} \mathrm{I} \quad(n \neq 2) \\ y^{\prime \prime}=\frac{3}{4} \frac{y^{\prime 2}}{y}+\left(b+\frac{c}{y}\right) y^{\prime}+e y^{2}+f y+g-\frac{c^{2}}{y}\end{array}\right.$ 
Équations différentielles dont l'intégrale générale est à points critiques fixes.

(4) $\quad y^{\prime \prime}=\frac{y^{\prime 2}}{y}\left(\mathrm{I}-\frac{\mathrm{I}}{n}\right)+\left(a y+b+\frac{c}{y}\right) y^{\prime}-\frac{n a^{2}}{(n+2)^{2}} y^{3}+e y^{2}+f y+g-\frac{n c^{2}}{(n-2)^{2}} \frac{\mathrm{I}}{y}$

(5) $\quad y^{\prime \prime}=\frac{y^{32}}{2 y}+(a y+b) y^{\prime}+\frac{a^{2}}{2} y^{3}+e y^{2}+f y$

(6) $\quad y^{\prime \prime}=\frac{y^{\prime 2}}{2 y}+(a y+b) y^{\prime}+\frac{a^{2}}{2} y^{3}+e y^{2}+f y+g+\frac{h}{y}$

(7) $\quad y^{\prime \prime}=\frac{y^{\prime 2}}{2 y}+b y^{\prime}+d y^{3}+e y^{2}+f y$

(8) $\quad y^{\prime \prime}=\frac{y^{\prime 2}}{2 y}+b y^{\prime}+d y^{3}+e y^{2}+f y+g+\frac{h}{y}$

(9) $\quad y^{\prime \prime}=\frac{y^{\prime 2}}{2 y}+b y^{\prime}+e y^{2}+f y+g+\frac{h}{y}$

(Io) $y^{\prime \prime}=\frac{y^{\prime 2}}{2 y}+(a y+b) y^{\prime}-\frac{a^{2}}{8} y^{3}+e y^{2}+f y+g+\frac{h}{y}$

(II) $\quad y^{\prime \prime}=\frac{2 y^{\prime 2}}{3 y}+(a y+b) y^{\prime}+\frac{3 a^{2}}{2} y^{3}+e y^{2}+f y$

(I2) $y^{\prime \prime}=\frac{2 y^{\prime 2}}{3 y}+\left(a y+b+\frac{c}{y}\right) y^{\prime}+\frac{3 a^{2}}{2} y^{9}+e y^{2}+f y+g-\frac{3 c^{8}}{y}$

(I3) $y^{\prime \prime}=\frac{4 y^{\prime 2}}{5 y}+(a y+b) y^{\prime}+5 a^{2} y^{3}+e y^{2}+f y$

(I4) $y^{\prime \prime}=\frac{4 y^{\prime 2}}{5 y}+\left(a y+b+\frac{c}{y}\right) y^{\prime}+5 a^{2} y^{3}+e y^{2}+f y+g-\frac{5 c^{2}}{9 y}$.

Sous cette forme on a déjà exprimé toutes les conditions provenant des simplifiées; il n'y a plus qu'à écrire celles qui correspondent aux zéros ou aux pôles mobiles. On verra plus loin que certains types dont les simplifiées sont distinctes s'étudient ensemble: telles les équations (5) et (6), ou encore (7) et (8), ou encore (II) et (I2), ou enfin (I3) et (I4).

Je ferai remarquer que l'équation

$$
y^{n}=\left(\mathrm{I}-\frac{\mathrm{I}}{n}\right) \frac{y^{\prime 2}}{y}+\left(a y+b+\frac{c}{y}\right) y^{\prime}+d y^{3}+e y^{2}+f y+g+\frac{h}{y}
$$

garde la même forme par la transformation $y=\lambda(x) Y, X=q(x)$, qui nous sera utile plus d'une fois pour établir entre les coefficients de cette équation une ou deux relations convenablement choisies. Les coefficients de la nouvelle équation $Y^{\prime \prime}=\left(\mathrm{I}-\frac{\mathrm{I}}{n}\right) \frac{Y^{\prime 2}}{Y}+\left(a_{1} Y+b_{1}+\frac{c_{1}}{Y}\right) Y^{\prime}+\cdots$ se calculent ainsi par les formules $\mathrm{F}$ 


$$
\left\{\begin{array}{l}
a_{1}=\frac{a \lambda}{\varphi^{\prime 2}}, \quad d_{1}=\frac{d \lambda^{2}}{\varphi^{\prime 1}}, \quad c_{1}=\frac{c}{\lambda \varphi^{\prime \prime}}, h_{1}=\frac{h}{\lambda^{2} \varphi^{\prime 2}} \\
b_{1}=\frac{b-\frac{2}{n} \frac{\lambda^{\prime}}{\lambda}-\frac{\varphi^{\prime \prime}}{\varphi^{\prime}}}{\varphi^{\prime}}, e_{1}=\frac{a \lambda^{\prime}+e \lambda}{\varphi^{\prime 2}}, g_{1}=\frac{c \frac{\lambda^{\prime}}{\lambda}+g}{\lambda \varphi^{\prime 2}} \\
f+b \frac{\lambda^{\prime}}{\lambda}-\frac{\mathrm{I}}{n}\left(\frac{\lambda^{\prime}}{\lambda}\right)^{2}-\frac{d}{d x}\left(\frac{\lambda^{\prime}}{\lambda}\right) \\
f_{1}=\frac{\frac{f 2}{\varphi^{\prime 2}}}{}
\end{array}\right.
$$

Dans ce qui suit j'indiquerai pour chaque type les équations qu'il donne au tableau $(T)$, l'intégration de cette équation, quand cette équation est intégrable, ou la réduction de cette équation à une équation du tableau $(t)$. Enfin j'indiquerai, quand ce ne sera pas trop long, les types correspondants du tableau $\Theta$, avec la substitution, qui permet de passer du type du tableau $\Theta$ au type correspondant du tableau $T$. C'est ici que doivent servir les formules (F).

Il sera bien entendu qu'une équation (E) sera réductible à l'un des types $T$ par une substitution $y=\hat{\lambda}(x) Y, X=\varphi(x)$ où $\lambda$ et $\varphi$ seront données par les opérations indiquées pour chacune: pour l'équation $T$ j'emploierai les lettres $X, Y$. Pour passer de l'équation $(\mathrm{E})$ au type $\Theta$ il suffira d'une substitution $y=\lambda(x) \eta, \xi=\varphi(x)$ où $\lambda$ et $\varphi$ se calculent algébriquement au moyen de $a, b, \ldots, h$. Comme ce calcul est extrêmement simple, je ne l'indiquerai pas et indiquerai tout simplement les opérations à faire pour passer du type $\Theta$ au type $T$ correspondant. On remarquera d'ailleurs que si le type $\Theta$ ou $T$ est intégrable, ces opérations coincident toujours avec celles que l'on doit faire pour intégrer le type $\Theta$. Les lettres $\alpha, \beta, \gamma, \delta$ désigneront des constantes numériques données, les lettres $q, r, s, \ldots$ des fonctions analytiques de la variable indépendante, $K$ une constante arbitraire.

5. Equations du premier type.

$$
Y^{\prime \prime}=\left(\mathrm{I}-\frac{\mathrm{I}}{n}\right) \frac{Y^{\prime 8}}{Y} \quad \eta^{\prime \prime}=\left(\mathrm{I}-\frac{\mathrm{I}}{n}\right) \frac{\eta^{\prime \mathbf{9}}}{\eta}+q(\xi) \eta^{\prime}+n r(\xi) \eta ;
$$

intégration: $Y=Z^{n}, \quad Z^{\prime \prime}=0 ; \eta=\zeta^{n}, \zeta^{\prime \prime}=q \zeta^{\prime}+r \zeta$. Connaissant deux intégrales particulières $\zeta_{1}$ et $\zeta_{2}$ de $\zeta^{\prime \prime}=q \zeta^{\prime}+r \zeta$ on posera $r_{\gamma}=\zeta^{n}(\xi) Y, X=\frac{\zeta_{2}(\xi)}{\zeta_{1}(\xi)}$ pour passer du type $\Theta$ au type $T$. Si l'on préfère, connaissant deux intégrales de l'équation $\Theta$, $\eta_{1}$ et $\eta_{2}$ on pose $\eta=\eta_{1}(\xi) Y, X=\sqrt[n]{\frac{\eta_{2}}{\eta_{1}}}$. Ici le type $(t)$ est $Y^{\prime \prime}=0$. 
Équations différentielles dont l'intégrale générale est à points critiques fixes.

(2)

$$
\begin{aligned}
& Y^{\prime \prime}=\frac{Y^{\prime 2}}{2 Y}+4 Y^{2} \quad \eta^{\prime \prime}=\frac{\eta^{\prime 2}}{2 \eta}-3 q \eta^{\prime}+4 \eta^{2}-2 \eta\left(q^{\prime}+2 q^{2}\right) ; \\
& Y=Z^{2}, \quad Z^{\prime \prime}=2 Z^{3} \text { d'où } Z=\frac{p\left(X_{1}-\mathrm{I}, \mathrm{o}\right)+\frac{\mathrm{I}}{2}}{p\left(X_{1}-\mathrm{I}, \mathrm{o}\right)-\frac{\mathrm{I}}{2}} \\
& \eta=\zeta^{2}, \quad \zeta=\frac{p(u,-\mathrm{I}, \mathrm{o})+\frac{\mathrm{I}}{2}}{p(u,-\mathrm{I}, \mathrm{o})-\frac{\mathrm{I}}{2}} \cdot u^{\prime}, \frac{u^{\prime \prime}}{u^{\prime}}=-q(\xi) .
\end{aligned}
$$

On passe de $\Theta$ à $T$ par $\eta=\varphi^{\prime 2}(\xi) Y, X=\varphi(\xi), \frac{\varphi^{\prime \prime}}{\varphi^{\prime}}=-q$.

(3) $\quad Y^{\prime \prime}=\frac{Y^{\prime 2}}{2 Y}+4 Y^{2}+2 Y \quad \eta^{\prime \prime}=\frac{\eta^{\prime 2}}{2 \eta}+\frac{q^{\prime}}{2 q} \eta^{\prime}+q\left(4 \eta^{2}+2 \eta\right)$;

$Y=Z^{2}, \quad Z^{\prime \prime}=2 Z^{3}+Z$, fonctions elliptiques, $d X=\frac{d Z}{\sqrt{Z^{4}+Z^{2}+K}} ;$

$\eta=Y, \quad d X=\sqrt{q(\xi)} d \xi$.

(4) $\quad Y^{\prime \prime}=\frac{Y^{\prime 2}}{2 Y}+4 Y^{2}+2 X Y, \quad Y=Z^{2}, \quad Z^{\prime \prime}=2 Z^{3}+X Z$ (trans. II).

(5) $\quad Y^{\prime \prime}=\frac{3 Y^{\prime 2}}{4 Y}+3 Y^{2} \quad \eta^{\prime \prime}=\frac{3 \eta^{\prime 2}}{4 \eta}+2 q \eta^{\prime}+3 \eta^{2}+\left(2 q^{\prime}-3 q^{2}\right) \eta$

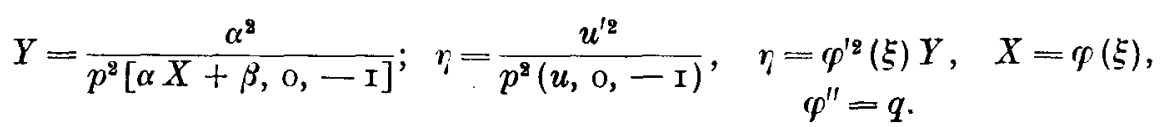

(6) $\quad Y^{\prime \prime}=\frac{3 Y^{\prime 8}}{4 Y}+3 Y^{9}+\alpha Y+\beta \quad \eta^{\prime \prime}=\frac{3}{4} \frac{\eta^{\prime 2}}{\eta}+\frac{q^{\prime}}{2 q} \eta^{\prime}+q\left(3 \eta^{2}+\alpha \eta+\beta\right) ;$

$$
\begin{aligned}
Y=Z^{8}, & Z^{\prime 2}=Z^{4}+\alpha Z^{2}+4 K Z-\beta, \quad \text { fonctions elliptiques; } \\
\eta=Y, & X=\varphi(\xi), \quad \varphi^{\prime 2}(\xi)=q .
\end{aligned}
$$

(7) $\quad Y^{\prime \prime}=\frac{3 Y^{\prime 2}}{4 Y}-\mathrm{I} \quad \eta^{\prime \prime}=\frac{3}{4} \frac{\eta^{\prime 2}}{\eta}+4 q \eta-\mathrm{I}$;

$\frac{\mathrm{I}}{\eta}=\left(\frac{u_{1}^{\prime}}{u_{1}}-\frac{u_{2}^{\prime}}{u_{2}}\right)^{2}, u_{1}$ et $u_{2}$ étant deux solutions de $u^{\prime \prime}=q u$.

On peut remarquer ici qu'en posant $\eta=\zeta^{2}$ on a $\zeta^{\prime \prime}=\frac{\zeta^{\prime 2}}{2 \zeta}+2 q \zeta-\frac{1}{2 \zeta}$ et qu'en différentiant $2 \zeta \zeta^{\prime \prime}=\zeta^{\prime 2}+4 q \zeta^{2}-I$ nous obtenons $\zeta^{\prime \prime \prime}=4 q \zeta^{\prime}+2 q^{\prime} \zeta$, équation linéaire du $3^{\text {ème }}$ ordre. Pour intégrer cette équation du $3^{\text {ème }}$ ordre nous remar- 
B. Gambier.

quons qu'elle admet l'intégrale première $2 \zeta \zeta^{\prime \prime}=\zeta^{\prime 2}+4 q \zeta^{2}+K$ où $K$ est une constante arbitraire; pour $K$ nul on a précisément l'équation $\zeta^{\prime \prime}=\frac{\zeta^{-12}}{2 \zeta}+2 q \zeta$ rencontrée au début de ce paragraphe de sorte que si $u_{1}$ et $u_{2}$ sont deux intégrales de $u^{\prime \prime}=q u,\left(K_{1} u_{1}+K_{2} u_{2}\right)^{2}$ satisfait à $\zeta^{\prime \prime \prime}=4 q \zeta^{\prime}+2 q^{\prime} \zeta$ donc $u_{1}^{2}$, $u_{2}^{2}$ et $u_{1} u_{3}$ sont trois intégrales de $\zeta^{\prime \prime \prime}=4 q \zeta^{\prime}+2 q^{\prime} \zeta$ qui se trouve ainsi intégrée par l'intermédiaire d'une équation différentielle du second ordre. C'est cette remarque qui conduit aussi aisément au résultat simple indiqué pour $\eta$.

Substitution de $\Theta$ à $T$ :

$$
\eta=u_{1}^{4} Y, X=\frac{u_{2}}{u_{1}}, \quad u_{1} \text { et } u_{2} \text { intégrales de } u^{n}=q u
$$

6. Equations du second type.

intégration:

$$
Y^{\prime \prime}=\left(\mathrm{I}-\frac{\mathrm{I}}{n}\right) \frac{Y^{\prime 2}}{Y}+Q Y Y^{\prime}-\frac{n Q^{2}}{(n+2)^{2}} Y^{3}+\frac{n Q^{\prime}}{(n+2)} Y^{2}
$$

$$
Y=\frac{\left(K_{1} X+K_{2}\right)^{n}}{u}, \quad u^{\prime}=\frac{-n Q}{n+2}\left(K_{1} X+K_{2}\right)^{n}
$$

type $\Theta$ :

$$
y^{n}=\left(\mathrm{I}-\frac{\mathrm{I}}{n}\right) \frac{y^{\prime 2}}{y}+q y y^{\prime}+r y^{\prime}-\frac{n q^{2}}{(n+2)^{2}} y^{3}+\frac{n}{n+2}\left(q^{\prime}-q r\right) y^{2}+n s y
$$

intégration:

d'où

$$
\frac{y^{\prime}}{y}-\frac{n q}{n+2} y=n z, \quad z^{\prime}+z^{2}-r z-s=0
$$

$$
y=\frac{v^{n}}{u}, \quad v^{\prime \prime}-r v^{\prime}-s v=0, \quad u^{\prime}=\frac{-n q}{n+2} v^{n}
$$

substitution de $\Theta$ à $T$ :

$$
y=v_{1}^{n} Y, \quad X=\frac{v_{2}}{v_{1}}
$$

$v_{1}$ et $v_{2}$ étant deux intégrales de $v^{\prime \prime}-r v^{\prime}-s v=0$.

(2) $\quad Y^{\prime \prime}=\frac{3}{4} \frac{Y^{\prime 2}}{Y}-\frac{3}{2} Y Y^{\prime}-\frac{Y^{3}}{4}+\frac{q^{\prime}}{2 q}\left(Y^{\prime}+Y^{2}\right)+r Y+q$;

$$
\begin{aligned}
& Y=\frac{q}{2 u^{\prime}+u^{2}-\frac{q^{\prime}}{q} u-r}, u=\frac{t^{\prime}}{t}, \\
& \quad t^{\prime \prime \prime}=\frac{3 q^{\prime}}{2 q} t^{\prime \prime}+\left(r+\frac{q^{\prime \prime}}{q}-\frac{q^{\prime 2}}{q^{8}}\right) t^{\prime}+\left(\frac{r^{\prime}}{2}+\frac{q}{2}-\frac{q^{\prime} r}{2 q}\right) t
\end{aligned}
$$

Ici le type $T$ et $\Theta$ coïncident. 
Équations différentielles dont l'intégrale générale est à points critiques fixes.

7. Equations du troisième type.

Pour $n$ quelconque ces équations rentrent dans le $4^{\text {ème }}$ type. Pour $n=4$ on a en plus des équations du cas général, où $n$ a été remplacé par 4 , l'équation

$$
\begin{array}{r}
Y^{\prime \prime}=\frac{3}{4} \frac{Y^{\prime 2}}{Y}+6 q^{\prime} \frac{Y^{\prime}}{Y}+3 Y^{2}+12 q Y-12 q^{\prime \prime}-\frac{36}{Y} q^{\prime 2} \text { avec } q^{\prime \prime}=6 q^{2}+S \\
\left(S=0, \frac{-1}{24} \text { ou } X\right) .
\end{array}
$$

Intégration:

$$
\left\{\begin{aligned}
3 Y & =2 V^{\prime}+V^{2}-\mathrm{I} 2 q, \quad V=\frac{Z^{\prime}-q^{\prime}}{Z-q}, Z^{\prime \prime}=6 Z^{2}+S \\
Y^{\prime} & =-\mathrm{I} 2 q^{\prime}-2 V Y
\end{aligned}\right.
$$

Ici, par exception avec ce qui suivra, j'explicite les équations du type $T$, et donne les équations correspondantes du type $\Theta$.

(I)

$$
\left\{\begin{array}{c}
Y^{\prime \prime}=\frac{3}{4} \frac{Y^{\prime 2}}{Y}-\frac{\mathrm{I} 2}{X^{3}} \frac{Y^{\prime}}{Y}+3 Y^{2}+\frac{\mathrm{I} 2 Y}{X^{2}}-\frac{7^{2}}{X^{4}}-\frac{\mathrm{I} 44}{X^{6} Y} \\
\eta^{\prime \prime}=\frac{3}{4} \frac{\eta^{\prime 2}}{\eta}+2\left(s+\frac{s^{\prime}}{s}\right) \eta^{\prime}-\mathrm{I} 2 s^{3} \frac{\eta^{\prime}}{\eta}+3 \eta^{2}+ \\
+\left(9 s^{2}-4 s^{\prime}-\frac{5 s^{18}}{s^{2}}+\frac{2 s^{\prime \prime}}{s}\right) \eta-24 s^{3}\left(2 s-\frac{s^{\prime}}{s}\right)-\frac{144 s^{6}}{\eta} \\
\eta=Y \varphi^{\prime 8}(\xi), X=\varphi(\xi), \frac{\varphi^{\prime}}{\varphi}=s .
\end{array}\right.
$$

2)

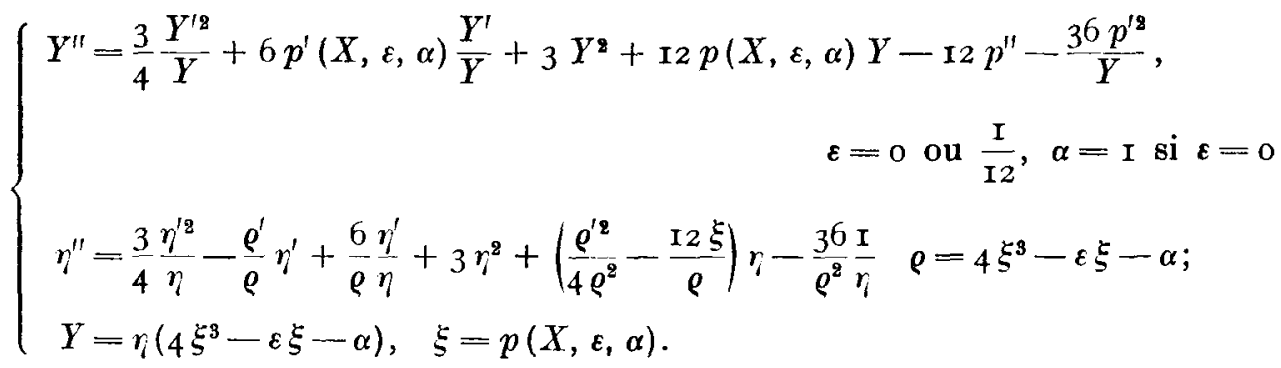

(3) $\quad Y^{\prime \prime}=\frac{3}{4} \frac{Y^{\prime 2}}{Y}+6 Q^{\prime} \frac{Y^{\prime}}{Y}+3 Y^{2}+12 Q Y-12 Q^{\prime \prime}-\frac{36 Q^{\prime 2}}{Y}, Q^{\prime \prime}=6 Q^{2}+X$.

Ici le type $T$ et $\Theta$ coïncident.

7. Equations du $4^{\text {ème }}$ type et $d u$ ro ème type (et $d u 3^{\text {̀̀me }}$ type).

Je consacre un chapitre spécial à ces équations. Je montrerai qu'elles doivent être réductibles à un type $T$ ou $\Theta$ : 


$$
\begin{gathered}
Y^{\prime \prime}=\left(\mathrm{I}-\frac{\mathrm{I}}{n}\right) \\
\frac{Y^{\prime 2}}{Y}+f_{n}(q, r) Y Y^{\prime}+\varphi_{n}(q, r) Y^{\prime}-\frac{n-2}{n} \frac{Y^{\prime}}{Y}-\frac{n f_{n}^{2}}{(n+2)^{2}} Y^{s} \\
+\frac{n\left[f_{n}^{\prime}-f_{n} \varphi_{n}\right]}{n+2} Y^{2}+\psi_{n}(q, r) Y-\varphi_{n}-\frac{\mathrm{r}}{n Y} .
\end{gathered}
$$

8. Equations $d u 5^{\text {ème }}$ et $d u 6^{\text {éme }}$ type.

Il est très simple d'écrire ici l'équation unique qui condense les nombreux types $T$.

$$
Y^{\prime \prime}=\frac{Y^{\prime 2}}{2 Y}-Y Y^{\prime}+q Y^{\prime}+\frac{Y^{8}}{2}-2 q Y^{2}+3\left(q^{\prime}+\frac{q^{2}}{2}\right) Y-\frac{72 r^{2}}{Y}
$$

où $r=\frac{V_{2}-V_{1}}{2}, q=\frac{V_{2}^{\prime}-V_{1}^{\prime}}{V_{2}-V_{1}} ; \quad V_{1}$ et $V_{2}$ sont deux intégrales particulières de $V^{\prime \prime}=6 V^{2}+S, \quad\left(S=0, \frac{-I}{24}\right.$, ou $\left.X\right)$.

L'intégration se fait en posant

d'où

$$
Y^{\prime}+Y^{2}-3 q Y=\mathrm{I} 2\left[V-\frac{V_{1}+V_{2}}{2}\right]
$$

$$
V^{\prime \prime}=6 V^{2}+S, \quad Y=\frac{6\left[V-V_{1}\right]\left[V-V_{2}\right]}{V^{\prime}-\frac{V_{1}^{\prime}+V_{2}^{\prime}}{2}-q\left[V-\frac{V_{1}+V_{2}}{2}\right]}
$$

L'énumération des types $T$ étant pratiquement impossible, celle des types $\Theta$ l'est à fortiori. Si $V_{2} \neq V_{1}, r \neq 0$; si $V_{2}=V_{1}, r=0$ et $q$ s'obtient en cherchant la limite du rapport $\frac{V_{2}^{\prime}-V_{1}^{\prime}}{V_{2}-V_{1}}$ quand $V_{2}$ tend vers $V_{1}$.

De même la formule qui donne $Y$ devient illusoire si $V$ est égal à $V_{1}$ ou à $V_{2}$ : on n'a qu'à chercher la limite de ce quotient si $V$ tend vers $V_{1}$.

9. Equations $d u 7^{\mathrm{ème}}$ et $d u 8^{\mathrm{ème}}$ type.

$$
Y^{\prime \prime}=\frac{Y^{\prime 2}}{2 Y}+\frac{3 Y^{3}}{2} \quad \eta^{\prime \prime}=\frac{\eta^{\prime 2}}{2 \eta}+2 q \eta^{\prime}+\frac{3 \eta^{3}}{2}+\left(q^{\prime}-\frac{3 q^{2}}{2}\right) \eta
$$

Intégration:

Substitution:

$$
Y=\frac{\alpha}{p(\alpha X+\beta, \mathrm{o}, \mathrm{I})}, \quad \eta=\frac{u^{\prime}}{p(u, \mathrm{o}, \mathrm{I})}, \frac{u^{\prime \prime}}{u^{\prime}}=q
$$

$$
\eta=\varphi^{\prime}(\xi) Y, \quad X=\varphi(\xi), \quad \frac{\varphi^{\prime \prime}}{\varphi^{\prime}}=q
$$


Équations différentielles dont l'intégrale générale est à points critiques fixes.

(2) $Y^{\prime \prime}=\frac{Y^{\prime 2}}{2 Y}+\frac{3 Y^{3}}{2}+4 \alpha Y^{2}+2 \beta Y-\frac{\gamma^{8}}{2 Y}$

Intégration :

$$
\eta^{\prime \prime}=\frac{\eta^{\prime 2}}{2 \eta}+\frac{q^{\prime}}{2 q} \eta^{\prime}+q\left[\frac{3 \eta^{3}}{2}+4 \alpha \eta^{2}+2 \beta \eta-\frac{\gamma^{8}}{2 \eta}\right]
$$

$$
Y^{\prime 8}=Y^{4}+{ }_{4}^{\alpha} Y^{3}+{ }_{4}^{\beta} Y^{2}+{ }_{4} K Y+\gamma^{8}
$$

Substitution :

$$
\frac{d \eta}{\sqrt{\eta^{4}+4 \alpha \eta^{3}+4 \beta \eta^{2}+4 K \eta+\gamma^{2}}}=\sqrt{q} d \xi
$$

$$
\eta=Y, \quad X=\varphi(\xi), \quad \varphi^{\prime 2}(\xi)=q(\xi) .
$$

$$
Y^{\prime \prime}=\frac{Y^{18}}{2 Y}+\frac{3 Y^{3}}{2}+4 X Y^{8}+2\left(X^{8}-\alpha\right) Y-\frac{\beta^{2}}{2 Y} \text {. }
$$

C'est la $4^{\text {eme }}$ transcendante irréductible; les types $T$ ou $\Theta$ coincident.

10. Equations du $9^{\mathrm{dme}}$ type.

$$
Y^{\prime \prime}=\frac{Y^{\prime \mathbf{8}}-\mathrm{I}}{2 Y} \quad \eta^{\prime \prime}=\frac{\eta^{\prime \mathbf{2}}-\mathrm{I}}{2 \eta}+2 q \eta .
$$

Equations rencontrées incidemment à propos de l'équation (7) du $\mathrm{r}^{\text {er }}$ type. Intégration: $\frac{\mathrm{I}}{\eta}=\frac{u_{1}^{\prime}}{u_{1}}-\frac{u_{2}^{\prime}}{u_{2}}, u_{1}$ et $u_{2}$ étant deux intégrales quelconques de $u^{\prime \prime}=q u$.

Si $u_{1}$ et $u_{2}$ sont deux intégrales déterminées $\eta_{j}=u_{1}^{\prime} Y, X=\frac{u_{2}}{u_{1}}$ fait passer de $\Theta$ à $T$.

(2) $\quad Y^{\prime \prime}=\frac{Y^{\prime 2}-\mathrm{I}}{2 Y}+4 Y^{2}+\alpha Y \quad \eta^{\prime \prime}=\frac{\eta^{\prime 2}}{2 \eta}+\frac{q^{\prime}}{2 q} \eta^{\prime}+q\left(4 \eta^{2}+\alpha y-\frac{\mathrm{I}}{\eta}\right)$.

Intégration:

$$
Y^{\prime 2}=4 Y^{3}+2 \alpha Y^{8}+{ }_{4} K Y+\mathrm{I}
$$

$$
\frac{d \eta}{\sqrt{4 \eta^{3}+2 \alpha \eta^{8}+4 K \overline{\eta+1}}}=\sqrt{q} d \xi \text {. }
$$

$$
\eta=Y, \quad X=\varphi(\xi), \quad \varphi^{\prime}(\xi)=V_{q}
$$

$$
Y^{\prime \prime}=\frac{Y^{\prime 8}}{2 Y}+4 \alpha Y^{2}-X Y-\frac{\mathrm{I}}{2 Y}, \quad \alpha \neq 0
$$

Intégration:

$$
\begin{gathered}
Y^{\prime}=-I+2 V Y, \quad 2 \alpha Y=V^{\prime}+V^{2}+\frac{X}{2}, \\
V^{\prime \prime}=2 V^{3}+X V-2 \alpha-\frac{I}{2} \quad \text { transcendante irréductible II. }
\end{gathered}
$$


11. Equations $d u \mathrm{II}^{\mathrm{èm} \theta}$ type et $d u \mathrm{I}^{\mathrm{dm} \theta}$ type.

Je condense encore en une seule équation les nombreuses équations de ce type; je désigne par $2 u^{3}+S u+T$ l'une quelconque, mais bien déterminée des 3 expressions $2 u^{3}, 2 u^{3}+\alpha u+\beta, 2 u^{3}+x q+\alpha$. Dans ces conditions le type $T$ est

(r) $Y^{\prime \prime}=\frac{2}{3} \frac{Y^{\prime 2}}{Y}-\frac{2}{3} Y Y^{\prime}+\frac{2 q}{3} Y^{\prime}+r \frac{Y^{\prime}}{Y}+\frac{2}{3} Y^{3}-\frac{\text { Io } q}{3} Y^{2}+$

où l'on a

$$
+\left(4 q^{\prime}+r+\frac{8 q^{2}}{3}\right) Y+2 q r-3 r^{\prime}-\frac{3 r^{2}}{Y}
$$

Intégration:

$$
q^{\prime \prime}=2 q^{3}+S q+T \quad r=-\frac{S}{3}-\frac{2}{3}\left(q^{\prime}+q^{2}\right)
$$

$$
\left.\begin{array}{l}
Y^{\prime}+Y^{2}-4 q Y+3 r=3[V-q] Y \\
Y=\frac{V^{\prime}-q^{\prime}+V^{2}-q^{2}}{V-q}
\end{array}\right\} V^{\prime \prime}=2 V^{3}+S V+T .
$$

Pour $V=q$, un calcul de limite donnera la valeur de $Y$ et par suite l'intégrale générale de l'équation de Riccati $Y^{\prime}+Y^{2}-4 q Y+3 r=0$. Nous sommes ramenés au type $(t)$ connu $V^{\prime \prime}=2 V^{3}+S V+T$. Je fais remarquer que le type $V^{\prime \prime}=2 V^{3}+X V+\alpha$ est la seconde équation irréductible. J'ai annoncé que pour certaines valeurs numériques des paramètres qui y figurent ces équations pouvaient admettre des intégrales particulières qui ne sont plus des transcendantes irréductibles. Ceci se présente ici et l'étude faite dans ce paragraphe d'équations secondaires dérivées de l'équation primaire $V^{\prime \prime}=2 V^{3}+X V+\alpha$ met ce fait en évidence. Quelle différence existe-t-il entre le type (II) et le type (I2)?: c'est que $r$ est nul pour II, non nul pour I2. On a à résoudre la question suivante: si $q$ est une intégrale de $q^{\prime \prime}=2 q^{3}+X q+\alpha$, la fonction $q^{\prime}+q^{2}+\frac{X}{2}$ est-elle nulle ou pas? Si on écrit $q^{\prime}+q^{2}+\frac{X}{2}=0$ on en déduit $q^{\prime \prime}+2 q q^{\prime}+\frac{\mathrm{r}}{2}=0$ d'où $q^{\prime \prime}-\left(2 q^{3}+X q-\frac{\mathrm{I}}{2}\right)=0$ donc pour $\alpha=-\frac{\mathrm{I}}{2}$ l'équation $q^{\prime \prime}=2 q^{3}+X q-\frac{\mathrm{I}}{2}$ admet une famille d'intégrales ne dépendant que d'une constante, qui ne sont plus que des transcendantes déjà connues, à savoir les intégrales de $q^{\prime}+q^{2}+\frac{X}{2}=0$.

Il y a ici une autre valeur remarquable pour $\alpha$, c'est $\alpha=0$ auquel cas $V=\mathrm{o}$ est une intégrale de $V^{\prime \prime}=2 V^{3}+X V$. Il y a intérêt à montrer que $\alpha=0$ et $\alpha=-\frac{\mathrm{r}}{2}$ se ramènent l'un à l'autre. Prenons en effet 


$$
y^{\prime \prime}=2 y^{3}+x y, z^{\prime \prime}=2 z^{3}+x z-\frac{\mathrm{I}}{2} \text {. }
$$

Je pose $y^{\prime}=u y$ d'où $2 y^{3}=u^{\prime}+u^{2}-x, \quad u^{\prime \prime}=2 u^{3}-2 x u+$ I. $\quad$ Or en posant $u=-V \sqrt[3]{2}, X=-x \sqrt[8]{2}$ on a $V^{\prime \prime}=2 V^{3}+X V-\frac{\mathrm{I}}{2}$. Les 2 types $\alpha=0, \alpha=-\frac{\mathrm{I}}{2}$ se correspondent donc bien. Comment se correspondent les deux groupes d'intégrales pour $y^{\prime \prime}=2 y^{3}+x y, \quad u^{\prime \prime}=2 u^{3}-2 x u+\mathrm{I}$ ? A chaque intégrale $u$ correspond pour $y$ l'une ou l'autre des racines carrées de $u^{\prime}+u^{2}-x$, qui se trouve être le carré d'une fonction uniforme de $x$. Pour toutes les intégrales particulières $u$ telles que $u^{\prime}+u^{2}-x=0$ on a $y=0$. - Inversement à chaque intégrale $y$ correspond pour $u$ une seule intégrale $\frac{y^{\prime}}{y}$; pour l'intégrale $y=0$ il $y$ a une infinité d'intégrales pour $u$, ̀̀ savoir celles de $u^{\prime}+u^{2}-x=0$. Il est très curieux de voir que l'étude des équations secondaires met en évidence ces valeurs remarquables des paramètres pour les équations irréductibles. Ceci avait déjà eu lieu pour l'équation $V^{\prime \prime}=2 V^{3}+X V+\alpha:(\alpha=0$ a été signalé par l'équation (3) du premier type $Y^{\prime \prime}=\frac{Y^{\prime 2}}{2 Y}+4 Y^{2}+2 X Y$ qui par $Y=Z^{2}$ donne $Z^{\prime \prime}=2 Z^{9}+X Z$; $\alpha=-\frac{I}{2}$ se trouve signalé encore par l'équation (3) du $9^{\text {em } \theta}$ type: $Y^{\prime \prime}=\frac{Y^{\prime g}}{2 Y}+$ $+4 \beta Y^{2}-X Y-\frac{I}{2 Y}$ qui, supposant $\beta \neq 0$ s'intègre par $Y^{\prime}=-\mathrm{I}+2 V Y$, $2 \beta Y=V^{\prime}+V^{2}+\frac{X}{2}$ et $V^{\prime \prime}=2 V^{3}+X V-\frac{I}{2}-2 \beta ;$ pour $\beta=0$ on retrouverait l'équation (I) du $9^{\text {ème }}$ type qui s'intègre tout simplement par $V^{\prime}+V^{a}+\frac{X}{2}=0$ ).

Nous retrouverons les mêmes particularités pour les types secondaires dérivant de l'équation irréductible IV.

12. Equations du treizième et quatorzième type.

Je condense encore les équations du type $T$ en désignant par $6 V^{2}+S$ les 3 expressions $6 V^{2}, 6 V^{2}-\frac{I}{24}, 6 V^{8}+X$.

(I) $\quad Y^{\prime \prime}=\frac{4}{5} \frac{Y^{\prime 8}}{Y}-\frac{2}{3} Y Y^{\prime}-\frac{q}{5} Y^{\prime}+r \frac{Y^{\prime}}{Y}+\frac{4}{5} Y^{9}+\frac{\text { I } 4 q}{5} Y^{8}+$ $+\left(r-3 q^{\prime}+\frac{6 q^{2}}{5}\right) Y-\frac{I}{3}\left(q r+5 r^{\prime}\right)-\frac{5 r^{2}}{9}$

$$
q=\frac{V_{2}^{\prime}-V_{1}^{\prime}}{V_{2}-V_{1}}, \quad r=\frac{72}{5} V_{1}+\frac{36}{5} V_{2}-\frac{9}{5}\left(\frac{V_{2}^{\prime}-V_{1}^{\prime}}{V_{2}-V_{1}}\right)^{2}
$$

$V_{1}$ et $V_{2}$ sont deux intégrales de $V^{\prime \prime}=6 V^{2}+S$. 
Intégration:

$$
\left\{\begin{array}{l}
Y^{\prime}+Y^{2}+3 q Y+\frac{5 r}{3}=-\frac{5}{2} Y[Z-q] \\
Y=\frac{Z^{\prime}-q^{\prime}}{Z-q}-\frac{1}{2}[Z+q] \\
Z^{\prime \prime}=-Z Z^{\prime}+Z^{3}-\mathrm{I}_{2} V_{1}(X) Z+\mathrm{I}_{2} V_{1}^{\prime}
\end{array}\right.
$$

L'intégrale de l'équation en $Z$ est $Z=\frac{V^{\prime}-V_{1}^{\prime}}{V-V_{1}}, V^{\prime \prime}=6 V^{2}+S$. Ici on a deux calculs de limite à faire, si $V_{2}$ tend vers $V_{1}$ ou quand $Z$ tend vers $q$.

\section{CHAPITRE II.}

Equations $y^{\prime \prime}=y^{12}\left(\frac{I}{2 y}+\frac{I}{y-I}\right)+B(y, x) y^{\prime}+C(y, x)$

1. L'équation doit être de la forme

$$
y^{\prime \prime}=y^{\prime 2}\left(\frac{I}{2 y}+\frac{I}{y-I}\right)+y^{\prime} \frac{P(y, x)}{y(y-I)}+\frac{Q(y, x)}{y(y-I)}
$$

où $P$ et $Q$ sont des polynômes en $y$. La transformation $y Y=1$ ne change pas la forme de l'équation et montre que $P$ est du $3^{\text {ème }}$ degré au plus en $y, Q$ du $5^{\text {əme }}$. Soit alors

$$
P(y, x) \equiv a(x)+y(\cdots), \quad Q(y, x) \equiv b(x)+y(\cdots)
$$

en faisant la substitution $x=x_{0}+\alpha X, y=\alpha Y$ on obtient la simplifiée $Y^{\prime \prime}=\frac{Y^{\prime 2}}{2 Y}+a_{0} \frac{Y^{\prime}}{Y}-\frac{b_{0}}{Y}$ qui est précisément l'une des équations étudiées au chapitre $I^{e r}$. On voit ainsi l'importance que présente la discussion des équations $y^{\prime \prime}=\frac{y^{\prime 2}}{y}\left(x-\frac{I}{n}\right)+\cdots$. Les résultats obtenus au chapitre $I^{\text {er }}$, paragraphe 2 , nous indiquent que le coefficient de $\frac{y^{\prime}}{y}$ est nul; donc $a\left(x_{0}\right)=0$, d'où $a(x) \equiv 0$.

Donc $P(y, x)$ n'a pas de terme constant en $y$, non plus que de terme en $y^{3}$ (la transformation $y Y=I$ dont il a été question, ou la simplifiée obtenue par $x=x_{0}+\alpha X, Y=\alpha y$ le montrent). 
Équations différentielles dont l'intégrale générale est à points critiques fixes.

Avec un changement de notations j'écris l'équation

$$
y^{\prime \prime}=y^{\prime 2}\left(\frac{\mathrm{I}}{2 y}+\frac{\mathrm{I}}{y-\mathrm{I}}\right)+y^{\prime} \frac{a y-b}{y-\mathrm{I}}+y(y-\mathrm{I})\left[e y+f+\frac{g}{y^{2}}+\frac{h}{y}+\frac{k}{(y-\mathrm{I})^{2}}+\frac{l}{y-\mathrm{I}}\right] \text {. }
$$

La substitution $y Y=$ I n'opère sur l'équation que l'échange suivant

$$
\begin{array}{llllllll}
a & b & e & f & g & h & k & l \\
b & a & g & h & e & f & k & k-l
\end{array}
$$

Si $g \equiv 0$ je forme une simplifiée par $x=x_{0}+\alpha X, y=\alpha^{2} Y, Y^{\prime \prime}=\frac{Y^{\prime 2}}{2 Y}-h_{0}$ et d'après les résultats du paragraphe 3 du Chapitre $\mathrm{I}^{\text {er }} h(x) \equiv 0$. De même $e \equiv 0$ entraîne $f \equiv 0$.

Il reste maintenant à former la simplifiée relative à la valeur I de $y$ : on l'obtient par $x=x_{0}+\alpha X, y-\mathrm{I}=\alpha Y$ d'où $Y^{\prime \prime}=\frac{Y^{\prime 2}}{Y}+\left(a_{0}-b_{0}\right) \frac{Y^{\prime}}{Y}+\frac{k_{0}}{Y^{\prime}}$, ce qui donne, d'après les résultats relatifs aux équations $y^{\prime \prime}=\frac{y^{\prime 2}}{y}+\cdots, 3$ cas possibles: $a-b \equiv k \equiv 0 ; a-b \equiv 0, k=\equiv ; a-b \equiv \equiv 0, k \equiv 0$.

2. L'étude des simplifiées ne donne rien de plus ici. Je n'entre pas dans le détail des résultats fournis par l'étude des valeurs $\infty$, o ou I de $y$. Ces résultats se simplifient d'ailleurs beaucoup en remarquant qu'une substitution $X=\varphi(x), y=Y$ ne change pas la forme de l'équation. On trouve ainsi 4 types canoniques seulement.

$$
Y^{\prime \prime}=Y^{\prime 2}\left(\frac{I}{2 Y}+\frac{I}{Y-I}\right) \quad \eta^{\prime \prime}=\eta^{\prime 2}\left(\frac{I}{2 \eta}+\frac{I}{\eta-I}\right)+q(\xi) \eta^{\prime}
$$

(2) $\left\{\begin{aligned} Y^{\prime \prime}=Y^{\prime 2}\left(\frac{\mathrm{I}}{2 Y}+\frac{\mathrm{I}}{Y-I}\right) & +Y(Y-\mathrm{I})\left[\alpha(Y-\mathrm{I})+\beta \frac{Y-\mathrm{I}}{Y^{\bar{z}}}+\frac{\gamma}{Y-\mathrm{I}}+\frac{\delta}{(Y-\mathrm{I})^{\mathrm{g}}}\right] \\ \eta^{\prime \prime}=\eta^{\prime 2}\left(\frac{\mathrm{I}}{2 \eta}+\frac{\mathrm{I}}{\eta-\mathrm{I}}\right) & +\frac{q^{\prime}(\xi)}{2 q(\xi)} \eta^{\prime}+ \\ & +q(\xi) \eta_{\eta}(\eta-\mathrm{I})\left[\alpha\left(r_{i}-\mathrm{I}\right)+\beta \frac{\eta-\mathrm{I}}{r_{i}^{2}}+\frac{\gamma}{\eta-\mathrm{I}}+\frac{\delta}{(\eta-\mathrm{I})^{9}}\right]\end{aligned}\right.$

(3) $\quad Y^{\prime \prime}=Y^{\prime 2}\left(\frac{\mathrm{I}}{2 Y}+\frac{\mathrm{I}}{Y-\mathrm{I}}\right)-\frac{Y^{\prime}}{X}+\frac{(Y-\mathrm{I})^{2}}{X^{2}}\left(\alpha Y+\frac{\beta}{Y}\right)+\gamma \frac{Y}{X}+\frac{\delta Y(Y+\mathrm{I})}{Y-\mathrm{I}}$

(4) $Y^{\prime \prime}=Y^{\prime 2}\left(\frac{\mathrm{I}}{2 Y}+\frac{\mathrm{I}}{\bar{Y}-\mathrm{I}}\right)+\frac{2(q Y+r)}{Y-\mathrm{I}} Y^{\prime}+\frac{(Y-I)^{2}}{2}\left(s^{2} Y-\frac{t^{2}}{Y}\right)+2\left[q^{2}-r^{2}-\left(q^{\prime}+r^{\prime}\right)\right] Y$ avec $2 q s-s=0, \quad 2 r t+t=0$. 
B. Gambier.

3. Pour la $3^{\text {ème }}$ équation, qui est l'une de nos équations irréductibles, le type $T$ suffit. Pour la dernière qui est réductible à une équation de RICcatr, je prends la forme $T$ ou $\Theta$ identiques. Les deux premières équations s'intègrent par les fonctions exponentielles et elliptiques. Pour la première on passe de $\Theta$ à $T \operatorname{par} \eta=Y, X=\varphi(\xi), \frac{\varphi^{\prime \prime}}{\varphi^{T}}=q(\xi)$; pour la seconde on passe de $\Theta$ à $T$ par $\eta=Y$, $X=\varphi(\xi), \rho^{\prime 2}(\xi)=q(\xi)$.

Intégration de (I):

$$
Y=T h^{2}(\alpha X+\beta), \eta=T h^{2} u, \frac{u^{\prime \prime}}{u^{\prime}}=q(\xi) .
$$

Intégration de (2):

$$
Y^{\prime 2}=Y(Y-\mathrm{I})^{2}\left[2 \alpha Y-\frac{2 \beta}{Y}-\frac{2 \gamma}{Y-\mathrm{I}}-\frac{\delta}{(Y-\mathrm{I})^{2}}+K\right] .
$$

Intégration de (4): l'équation (4) équivaut à

$$
\left\{\begin{array}{l}
\frac{y^{\prime}+2(q+r)}{y-\mathrm{I}}-s y=-2 u \\
\frac{y-\mathrm{I}}{y}=-\frac{u^{\prime}+2 r u}{u^{2}-\frac{t^{2}}{4}} .
\end{array}\right.
$$

L'élimination de $y$ donne

$\frac{d}{d x}\left(u^{\prime}+2 r u\right)=-2(u+2 r)\left(u^{\prime}+2 r u\right)+2(q+r)\left[u^{\prime}+u^{2}+2 r u-\frac{t^{2}}{4}\right]+s\left(u^{\prime}+2 r u\right)$.

L'équation en $u$ rentre dans les équations $y^{\prime \prime}=B(y, x) y^{\prime}+C(y, x)$. On l'intègre en écrivant

$$
u^{\prime}+u^{2}+2 r u-\frac{t^{2}}{4}=\frac{z}{2}+s u, \quad \frac{z^{\prime}}{z}=2(q-r)
$$

ce qui donne une quadrature, suivie d'une équation de Riccati pour avoir $u$ et par suite $y$.

On remarquera que si ni $s$ ni $t$ ne sont nuls, la quadrature est toute faite, car on a alors $\frac{z^{\prime}}{z}=\frac{s^{\prime}}{s}+\frac{t^{\prime}}{t}$ ou $z=K t s$. On peut remarquer aussi que si $u= \pm \frac{t}{2}$ la formule qui donne $y$ est illusoire; il faut alors résoudre l'équation de Riccati

$$
y^{\prime}+2(q+r)=(s y \mp t)(y-\mathrm{I})
$$




\section{CHAPITRE III.}

Equations $y^{\prime \prime}=\frac{2}{3}\left(\frac{\mathrm{I}}{y}+\frac{\mathrm{I}}{y-\mathrm{I}}\right) y^{\prime 2}+\frac{P(y, x)}{y(y-\mathrm{I})} y^{\prime}+\frac{Q(y, x)}{y(y-\mathrm{I})}$.

1. - $P$ et $Q$ sont des polynômes en $y$, dont la substitution $y Y=I$ donne le degré: 3 pour $P, 5$ pour $Q$. J'écris donc l'équation

$$
\begin{aligned}
y^{\prime \prime}=\frac{2}{3}\left(\frac{\mathrm{I}}{y}+\frac{\mathrm{I}}{y-\mathrm{I}}\right) y^{\prime 2}+\left(a y+\frac{b}{y}-\frac{c}{y-\mathrm{I}}+d\right) y^{\prime}+ & \\
& \quad+y(y-\mathrm{I})\left[e y+\frac{f}{y^{2}}-\frac{g}{(y-\mathrm{I})^{2}}+h+\frac{k}{y}+\frac{l}{y-\mathrm{I}}\right] .
\end{aligned}
$$

Les valeurs o, $\mathrm{I}, \infty$ de $y$ jouent ici le même rôle. Si je fais par exemple $x=X$, $y=\mathrm{I}-Y$ à la valeur $y=\mathrm{I}$ correspond $Y=0$, la forme de l'équation n'est pas modifiée, il y a seulement les échanges suivants:

$$
\begin{array}{cccccccccc}
a & b & c & d & e & f & g & h & k & l \\
-a & c & b & a+d & e & g & f & e-h & l & k
\end{array}
$$

De même par $x=X, y Y=\mathrm{I}$ à $y=\infty$ correspond $Y=0$, la forme de l'équation n'est pas modifiée, sauf les échanges

$$
\begin{array}{cccccccccc}
a & b & c & d & e & f & g & h & k & l \\
b & a & -c & d+c & f & e & g & k & h & -l-g .
\end{array}
$$

Il suffit done d'étudier une seule des 3 valeurs $0, I, \infty$. Formons par exemple les simplifiées relatives à $y=0$. C'est la substitution $x=x_{0}+\alpha X, y=\alpha Y$ qui donne

$$
Y^{\prime \prime}=\frac{2}{3} \frac{Y^{\prime z}}{Y}+b_{0} \frac{Y^{\prime}}{Y}-\frac{f_{0}}{Y}
$$

C'est une équation du type $y^{\prime \prime}=\left(\mathrm{I}-\frac{\mathrm{I}}{n}\right) \frac{y^{\prime 2}}{y}+\cdots$ où $n=3$ et l'on sait d'après les résultats obtenus au chapitre $\mathrm{I}^{\mathrm{er}}$, paragraphe 2 , que ou bien $b_{0}=f_{0}=0$, ou $f_{0}=3 b_{0}^{2}$, c'est-ì-dire ou bien $b \equiv f \equiv 0$, ou bien $f(x) \equiv b^{\mathbf{2}}(x)$.

Si $b \equiv f \equiv 0$, on forme une seconde simplifiée par $x=x_{0}+\alpha X, y=\alpha^{2} Y$ qui est $Y^{\prime \prime}=\frac{2}{3} \frac{Y^{\prime 2}}{Y}-k_{0}$ et d'après les résultats du chapitre I, paragraphe $3, k_{0}=0$ ou $k \equiv 0$. 
On en déduit immédiatement que ou bien $a \equiv e \equiv h \equiv 0$ ou bien $e \equiv 3 a^{2}$ et de même ou bien $c \equiv g=l \equiv$ o ou bien $g \equiv 3 c^{8}$.

L'interprétation de $b \equiv f \equiv k \equiv 0$ est très simple: si l'on fait $y=z^{9}$ on a une équation $\mathrm{du}$ second ordre et du premier degré en $z^{\prime \prime}$ où la valeur $z=0$ est une valeur ordinaire; donc $b \equiv f \equiv k \equiv 0$ entraîne l'existence d'une famille de zéros triples mobiles.

Les résultats précédents conduisent à une équation obtenue en supposant $a \equiv b \equiv c \equiv 0, y^{\prime \prime}=\frac{2}{3}\left(\frac{I}{y}+\frac{I}{y-I}\right) y^{\prime g}+q(x) y^{\prime}$ qui est à points critiques fixes: en posant $y=Y, X=\varphi(x), \frac{\varphi^{\prime \prime}}{\varphi^{\prime}}=q$ on obtient $Y^{\prime \prime}=\frac{2}{3}\left(\frac{I}{Y}+\frac{I}{Y-I}\right) Y^{\prime 2}$ dont l'intégrale générale est $Y=\frac{p^{\prime}(\alpha X+\beta, 0,-I)+I}{2}$, de sorte que l'intégrale générale de $y^{\prime \prime}=\frac{2}{3}\left(\frac{\mathrm{I}}{y}+\frac{\mathrm{I}}{y-\mathrm{I}}\right) y^{\prime 2}+q(x) y^{\prime}$ est $y=\frac{p^{\prime}(u, \mathrm{o},-\mathrm{I})+\mathrm{I}}{2}$ avec $\frac{u^{\prime \prime}}{u^{\prime}}=q . \quad$ Il est facile de vérifier que dans le cas actuel $y, \mathrm{I}-y, \frac{I}{y}$ sont des cubes de fonctions à points critiques fixes.

2. - Si nous supposons maintenant que $a, b, c$ ne sont pas nuls tous 3 , il faudra avoir recours aux développements correspondant soit aux pôles, soit aux zéros, soit aux unités de $y$. Je fais remarquer que la substitution $y=Y$, $X=\varphi(x)$ ne change pas la forme de l'équation et remplace $d$ par $\left(d-\frac{\varphi^{\prime \prime}}{\varphi^{\prime}}\right) \frac{\mathrm{I}}{\varphi^{\prime}}$; $a, b, c \operatorname{par} \frac{a}{\varphi^{\prime}}, \frac{b}{\varphi^{\prime}}, \frac{c}{\varphi^{\prime}} ; e, f, \ldots l \operatorname{par} \frac{e}{\varphi^{\prime 2}}, \ldots \frac{l}{\varphi^{\prime z}} \cdot$ Cecj nous servira plus tard.

Puisque d'autre part $y Y=\mathrm{I}$, ou $y+Y=I$ ne change pas non plus la forme de l'équation, il est clair qu'une substitution $X=\varphi(x), y=\varrho_{i}(Y)$ où $\varrho_{i}$ est l'une des 6 fractions $\varrho_{1}=Y, \varrho_{2}=\frac{\mathrm{I}}{Y}, \varrho_{3}=\mathrm{I}-Y, \varrho_{1}=\frac{Y-I}{Y}, \varrho_{5}=\frac{\mathrm{I}}{\mathrm{I}-Y}, \varrho_{6}=\frac{Y}{Y-I}$ ne change pas non plus la forme de l'équation. Il me suffira donc d'indiquer, pour le tableau $T$, toutes les formes que l'on peut avoir en supposant $c \neq 0$, car du moment que $a, b, c$ ne sont pas nuls tous 3 et que $c$ peut être par l'une de ces substitutions échangé avec $b$, ou $a$, le cas de $c \neq 0$ suffira.

Formons les conditions relatives aux pôles si $a \neq 0$. Il y a alors deux familles de pôles simples: la première $y=\frac{-1}{3 a_{0} x-x_{0}}+\lambda+\cdots$ où $\lambda$ sera arbitraire, avec la relation $h+3\left(a d-a^{\prime}+a^{2}\right)=0$; la seconde famille de pôles est $y=\frac{2}{3 a_{0}} \frac{I}{x-x_{0}}+a_{1}+\lambda\left(x-x_{0}\right)+\cdots$ où $\lambda$ est arbitraire avec la relation $\left(z h+3 a^{2}\right)^{9}$ $+6\left(a h^{\prime}-2 h a^{\prime}\right)-9 a^{3}(b-c)+6 a^{2}(k+l)=0$. On remarque en passant que si 
Équations différentielles dont l'intégrale générale est à points critiques fixes.

$a(x) \equiv e(x) \equiv h(x) \equiv 0$, ces relations sont encore rérifiées, mais entraînent pour les pôles des conséquences différentes (une seule famille de pôles triples). Ces résultats s'étendent aux zéros et aux unités et on est done conduit à écrire l'équation

(I) $y^{\prime \prime}=\frac{2}{3}\left(\frac{\mathrm{I}}{y}+\frac{\mathrm{I}}{y-\mathrm{I}}\right) y^{\prime \mathbf{y}}+\left(a y+\frac{b}{y}-\frac{c}{y-\mathrm{I}}+d\right) y^{\prime}+$

$$
+y(y-\mathrm{I})\left[3 a^{2} y+\frac{3 b^{2}}{y^{2}}-\frac{3 c^{2}}{(y-\mathrm{I})^{2}}+h+\frac{k}{y}+\frac{l}{y-\mathrm{I}}\right]
$$

avec le système de relations différentielles algébriques

(2) $\left\{\begin{array}{l}h+3\left(a d+a^{2}-a^{\prime}\right)=0 \\ k+3\left(b d+b c+b^{2}-b^{\prime}\right)=0 \\ l+3\left(c a+c b+c d+c^{2}+c^{\prime}\right)=0\end{array}\right.$

(3) $\left\{\begin{array}{l}\left(2 h+3 a^{8}\right)^{2}+6\left(a h^{\prime}-2 h a^{\prime}\right)-9 a^{3}(b-c)+6 a^{2}(k+l)=0 \\ \left(2 k+3 b^{8}\right)^{2}+6\left(b h^{\prime}-2 k b^{\prime}\right)-9 b^{3}(a+c)+6 b^{2}\left(h-l-3 c^{2}\right)=0 \\ \left(2 l+3 c^{8}\right)^{2}+6\left(c l^{\prime}-2 l c^{\prime}\right)-9 c^{3}(b-a)-6 c^{2}\left(h+k+3 a^{9}+3 b^{2}\right)=0\end{array}\right.$

que j'ai cité comme type dans l'introduction, paragraphe 7 .

Je vais profiter de cet exemple, pour montrer la méthode qui permet d'intégrer les relations (2), (3) et qui démontre qu'alors ( $\mathrm{x}$ ) a ses points critiques fixes, donnant l'intégration de $(I)$ si elle est intégrable ou la ramenant à un type déjà connu. J'ai dit aussi que si l'on prenait maladroitement le système (2), (3) on no pouvait rien en tirer; or pour les calculs qui suivent je vais montrer que les 3 relations (3) sont inutiles, elles sont d'ailleurs assez difficiles à former, les relations (2) au contraire très simples, parce que le rang du coefficient indéterminé dans le développement polaire est moins élevé pour la $I^{\text {əre }}$ relation (2) que pour la $I^{\text {ère }}$ relation (3). Cette remarque d'ailleurs est générale.

3. Je prends, en supposant $a(x) \equiv \equiv$, le pôle mobile $y=\frac{-\mathrm{I}}{3 a_{0}} \frac{\mathrm{I}}{x-x_{0}}+\lambda+\cdots$, l'expression $\frac{y^{\prime}}{y}-3 a y$ est évidemment régulière pour un tel pôle, et la valeur qu'elle prend pour ce pôle est arbitraire au même titre que $\lambda$, à savoir $a_{0}^{\prime}-6 \lambda a_{0}$. Un pôle de la seconde famille $y=\frac{2}{3 a_{0}} \frac{I}{x-x_{0}}+\cdots$ est pour cette expression un pôle simple de résidu - 3. De même l'expression $\frac{y^{\prime}+3 b}{y}$, si $b=0$, est régulière pour le zéro mobile $y=-3 b_{0}\left(x-x_{0}\right)+\lambda\left(x-x_{0}\right)^{2}+\cdots$, la valeur qu'elle prend 
alors étant arbitraire; l'autre zéro mobile $y=\frac{3 b_{0}}{2}\left(x-x_{0}\right)+\cdots$ est pour cette expression un pôle mobile de résidu +3 . Je condense ces résultats en posant

$$
u=\frac{y^{\prime}+3 b-3 a y^{2}}{3 y}+A(x)
$$

où $A(x)$ est une fonction arbitraire; le premier pôle et le premier zéro de $y$ laissent $u$ régulière avec une valeur arbitraire; le second pôle ou zéro de $y$ sont pour $u$ un pôle de résidu $-\mathrm{I}$ ou $+\mathbf{I}$. Je choisis, pour la commodité des calculs $A \equiv \frac{c}{2}+a-b$. Je vérifie aisément, en me servant des relations (2) que l'équation (1) équivaut au système

(4)

$$
\left\{\begin{aligned}
u & =\frac{y^{\prime}+3 b-3 a y^{2}}{3 y}+\frac{c}{2}+a-b \\
y-I & =\frac{2 u\left(u-\frac{3 c}{2}\right)}{u^{\prime}-u^{8}-\alpha u+\beta},
\end{aligned}\right.
$$

où, pour abréger,

$$
\begin{aligned}
& \alpha \equiv 2 b+2 a-c+d \\
& \beta \equiv b^{\prime}-\frac{c^{\prime}}{2}+2 a c-\frac{k}{3}-\frac{l}{3}-d\left(b-\frac{c}{2}\right)-\left(b-\frac{c}{2}\right)^{2}
\end{aligned}
$$

Nous éliminons $y$ entre les équations (4): par exemple en remarquant que la $I^{\text {ere }}$ peut s'écrire

$$
\frac{y^{\prime}}{y-\mathrm{r}}=\frac{3\left(u-\frac{c}{2}\right)}{y-\mathrm{I}}+3\left(a+b-\frac{c}{2}+u\right)+3 a(y-\mathrm{I})
$$

On obtient ainsi

(5) $\frac{u^{\prime}}{u}+\frac{u^{\prime}-\frac{3 c^{\prime}}{2}}{u-\frac{3 c}{2}}=\frac{u^{\prime \prime}-2 u u^{\prime}-\alpha^{3} u-\alpha u^{\prime}+\beta^{\prime}}{u^{\prime}-u^{2}-\alpha u+\beta}+\frac{3\left(u-\frac{c}{2}\right)}{2 u\left(u-\frac{3 c}{2}\right)}\left(u^{\prime}-u^{2}-\alpha u+\beta\right)+$

$$
+3\left(a+b-\frac{c}{2}+u\right)+\frac{6 a u\left(u-\frac{3 c}{2}\right)}{u^{\prime}-u^{2}-\alpha u+\beta} .
$$


Équations différentielles dont l'intégrale générale est à points critiques fixes.

Si l'on remarque que

$$
\frac{3\left(u-\frac{c}{2}\right)}{2 u\left(u-\frac{3 c}{2}\right)}=\frac{\mathrm{I}}{2 u}+\frac{\mathrm{I}}{u-\frac{3 c}{2}}
$$

on voit immédiatement qu'en multipliant par $u^{\prime}-u^{2}-\alpha u+\beta$ les 2 membres do l'équation, on obtient

$$
u^{\prime \prime}=\frac{u^{\prime z}}{2 u}+P(x, u) u^{\prime}+Q(x, u)
$$

donc $P$ et $Q$ ne peuvent, admettre $u-\frac{3 c}{2}$ comme pôle: il suffit donc, avant multiplication, d'écrire que dans (5) $u-\frac{3 c}{2}$ n'existe pas en dénominateur ou que le polynôme en $u$ suivant, $u^{\prime}-\frac{3 c^{\prime}}{2}-\left(u^{\prime}-u^{2}-\alpha u+\beta\right)$, est divisible par $u-\frac{3 c}{2}$, ce qui donne la relation $\beta=\frac{3 c}{2}\left(\alpha+\frac{3 c}{2}\right)-\frac{3 c^{\prime}}{2}$. On vérifierait aisément que cette relation est une conséquence des relations (3): or mon but est de prouver qu'il est inutile de former ces relations (3), et que le cours des calculs doit les donner, ou plutôt donner 3 équations équivalentes, où les coefficients sont mieux groupés. Cette relation admise nous avons

(6) $u^{\prime \prime}=\frac{u^{\prime 2}}{2 u}+(a+b+c+2 d) u^{\prime}+\frac{3 u^{3}}{2}+3(b-a-c) u^{2}-$

$$
-\left[\frac{3 \alpha(c+d)}{2}+\beta-\alpha^{\prime}-9 a c\right] u+\beta(a+b+c+2 d)-\beta^{\prime}-\frac{\beta^{2}}{2 u} .
$$

Cette équation (6) résoud la question: nous l'avons étudiée, chapitre ${ }^{\mathrm{er}}$, paragraphe 9. Nous savons même, qu'il y a avantage pour avoir les types réduits, à supposer $a+b+c+2 d=0$; on $y$ arrive par $y=Y, X=\varphi(x), u=V \varphi^{\prime}(x)$, substitution qui laisse intacte les relations (4), où $y$ et $u$ sont remplacées par $Y$ et $V$, les coefficients de ( $\mathrm{r}$ ) étant remplacés par ceux de l'équation en $Y$ et $X$.

Il suffit alors de déterminer $\varphi$ par la relation $\frac{\varphi^{\prime \prime}}{\varphi^{\prime}}=\frac{a+b+c+2 d}{2}$, ce qui revient à ajouter aux 6 relations (2) et (3) la $7^{\text {èm } \theta} a+b+c+2 d=0$, difficile à prévoir à priori et qui va permettre de déterminer les 7 fonctions $a, b, c, d, h, k, l$ des types $T$; il suffit d'ailleurs de déterminer $a, b, c, d$ en tenant compte des 3 relations (2). Il n'y a plus alors qu'à écrire que l'équation (6) coïncide, moyennant $a+b+c+2 d=0$ avec l'équation 
B. Gambier.

(7)

$$
V^{\prime \prime}=\frac{V^{\prime 8}}{2 V}+\frac{3}{2} V^{3}+{ }_{4} C V^{2}+2 D V-\frac{E^{2}}{2 V}
$$

où le second membre désigne soit $\frac{V^{\prime 2}}{2 V}+\frac{3}{2} V^{3}$, soit $\frac{V^{\prime 8}}{2 V}+\frac{3}{2} V^{3}+4 \alpha V^{3}+2 \beta V-$ $\frac{\gamma^{2}}{2 V}$ soit $\frac{V^{\prime 2}}{2 V}+\frac{3}{2} V^{2}+4 X V^{2}+2\left(X^{2}-\alpha\right) V-\frac{\beta^{3}}{2 V}, \alpha, \beta, \gamma$ étant des constantes numériques. Cela donne des équations différentielles très simples, donnant sans effort $a, b, c, d$.

4. - Je donne le simple résultat: le tableau $T$ se réduit (moyennant une substitution auxiliaire $Y=\varrho_{i}\left(Y_{1}\right)$, dont il a été parlé, si c'est nécessaire) aux 2 équations

$$
Y^{\prime \prime}=\frac{2}{3}\left(\frac{I}{Y}+\frac{I}{Y-I}\right) Y^{\prime 2} \quad \eta^{\prime \prime}=\frac{2}{3}\left(\frac{I}{\eta}+\frac{I}{\eta-I}\right) \eta^{\prime 8}+q(\xi) \eta
$$

(2) $Y^{\prime \prime}=\frac{2}{3}\left(\frac{\mathrm{Y}}{Y}+\frac{\mathrm{I}}{Y-\mathrm{I}}\right) Y^{\prime 2}+\left(q Y+\frac{r}{Y}-\frac{s}{Y-\mathrm{I}}-\frac{q+r+s}{2}\right) Y^{\prime}$

$$
\begin{array}{r}
+Y(Y-\mathrm{I})\left[3 q^{2} Y+\frac{3 r^{2}}{Y^{2}}-\frac{3 s^{2}}{(Y-1)^{2}}+3 q^{\prime}+\frac{3 q}{2}(r+s+q)+\right. \\
\left.+\frac{3 r^{\prime}-\frac{3 r}{2}(r+s-q)}{Y}+\frac{3 s^{\prime}-\frac{3 s}{2}(q+r+s)}{Y-I}\right]
\end{array}
$$

avec

$$
\begin{aligned}
& 3 q=\frac{V_{1}^{\prime}}{V_{1}}-V_{1}+\frac{E}{V_{1}}-2 C, \quad 3 r=\frac{V_{1}^{\prime}}{V_{1}}+V_{1}+\frac{E}{V_{1}}+2 C, \\
& 3 s=2 V_{1}, \quad V_{2}^{\prime \prime}=\frac{V_{1}^{\prime 2}}{2 V_{1}}+\frac{3 V_{1}^{3}}{2}+4 C V_{1}^{2}+2 D V_{1}-\frac{E^{3}}{2 V_{1}} .
\end{aligned}
$$

Intégration:

$$
\begin{aligned}
& V=\frac{Y^{\prime}+3 r-3 q Y^{2}}{3}+\frac{s}{2}+q-r, \\
& \qquad Y-\mathrm{I}=\frac{2 V\left(V-V_{1}\right)}{V^{\prime}-V_{1}^{\prime}-\left(V^{2}-V_{1}^{2}\right)-\frac{3}{2}(q+r-s)\left(V-V_{1}\right)}, \\
& V^{\prime \prime}=\frac{V^{\prime \mathbf{g}}}{2 V}+\frac{3}{2} V^{3}+4 C V^{2}+2 D V-\frac{E^{\mathrm{e}}}{2}, \\
& \text { où } C, D, E \text { sont ou bien nulles toutes } 3 \text {, ou égales à } 3 \text { constantes non nulles } \\
& \text { ensemble, ou bien égales } C \text { à } X, D \text { à } X^{2}-\alpha \text { et } E \text { constante. }
\end{aligned}
$$


Équations différentielles dont l'intégrale générale est à points critiques fixes.

5. - Je fais encore remarquer ici que les types dérivés de l'équation irréductible $Y^{\prime \prime}=\frac{Y^{\prime 2}}{2 Y}+\frac{3 Y^{9}}{2}+4 X Y^{\mathrm{s}}+2\left(X^{3}+\alpha\right) Y-\frac{\beta^{8}}{2 Y}$ mettent encore en évidence des valeurs particulières de $\alpha$ et $\beta$ pour lesquelles des intégrales particulières de cette équation sont des transcendantes banales. Il suffit de chercher si $q$ ou $r$ peut être nul, c'est-à-dire si les intégrales de l'une ou l'autre équation de RICOATI $Y^{\prime}+Y^{2}+2 X Y+\beta=0$ ou $Y^{\prime}-Y^{2}-2 X Y+\beta=0$ peuvent satisfaire à l'équation $Y^{\prime \prime}=\frac{Y^{\prime 2}}{2 Y}+\frac{3 Y^{3}}{2}+4 X Y^{2}+2\left(X^{2}-\alpha Y-\frac{\beta^{2}}{2 Y}\right.$; on trouve que si $\beta=$ $2(\mathrm{I}-\alpha)$ toutes les solutions de la première équation de Rrccati sont solutions et si $\beta=2(\mathrm{I}+\alpha)$ toutes les intégrales de la seconde équation de RICcati sont solutions.

\section{CHAPITRE IV.}

$$
\text { Equations } y^{\prime \prime}=\frac{3}{4}\left(\frac{I}{y}+\frac{I}{y-1}\right) y^{\prime 2}+B(y, x) y^{\prime}+C(y, x)
$$

1. On voit aisément que l'équation est de la forme

$$
\begin{aligned}
y^{\prime \prime}=\frac{3}{4}\left(\frac{\mathrm{I}}{y}+\frac{\mathrm{I}}{y-\mathrm{I}}\right) y^{\prime 2}+\left(a+\frac{b}{y}-\frac{c}{y-\mathrm{I}}\right) y^{\prime}+ & \\
& +y(y-\mathrm{I})\left[4 d^{2}(2 y-\mathrm{I})+\frac{b^{2}}{y^{2}}-\frac{c^{2}}{(y-\mathrm{I})^{8}}+\frac{h}{y}+\frac{k}{y-\mathrm{I}}\right]
\end{aligned}
$$

aveo $d a-d^{\prime}=0$. La transformation $x=X, y+Y=\mathrm{I}$ laisse la forme de l'équation invariable sauf échange de $b$ et $c, h$ et $k$. J'ai à inscrire au tableau $T 3$ classes d'équations.

2. D'abord deux équations qui s'intègrent par les fonctions elliptiques

$$
Y^{\prime \prime}=\frac{3}{4}\left(\frac{I}{Y}+\frac{I}{Y-I}\right) Y^{\prime 2} \quad \eta^{\prime \prime}=\frac{3}{4}\left(\frac{I}{\eta}+\frac{\mathrm{I}}{\eta-I}\right) \eta^{\prime 2}+q(\xi) \eta^{\prime}
$$

Intégration:

$$
Y=\frac{\mathrm{I}}{\mathrm{I}-p^{2}(\alpha X+\beta, 0,4)} ; \quad \eta=\frac{\mathrm{I}}{\mathrm{I}-p^{2}(u, 0,4)}, \frac{u^{\prime \prime}}{u}=q(\xi) .
$$


B. Gambier.

(2)

$$
Y^{\prime \prime}=\frac{3}{4}\left(\frac{\mathrm{I}}{Y}+\frac{\mathrm{I}}{\bar{Y}-\mathrm{I}}\right) Y^{\prime 2}+Y(Y-\mathrm{I})\left[\frac{\alpha}{\bar{Y}}+\frac{\beta}{Y-\mathrm{I}}+2 \gamma(Y-\mathrm{I})\right] .
$$

Intégration:

$$
Y=\frac{\mathrm{I}}{\mathrm{I}-u^{2}}, \quad u^{\prime 2}=\left[\alpha u-\frac{\beta}{u}-\frac{\gamma}{u-\mathrm{I}}+K\right] u\left(\mathrm{I}-u^{2}\right) .
$$

Le type $\Theta$ est

$$
\eta^{\prime \prime}=\frac{3}{4}\left(\frac{\mathrm{I}}{\eta}+\frac{\mathrm{I}}{\eta-\mathrm{I}}\right) \eta^{\prime 2}+\frac{q^{\prime}(\xi)}{2 q(\xi)} \eta^{\prime}+q(\xi)\left[\frac{\alpha}{\eta}+\frac{\beta}{\eta-\mathrm{I}}+2 \gamma(2 \eta-\mathrm{I})\right]
$$

Substitution:

$$
\eta=Y, \quad X=\varphi(\xi), \quad \varphi^{\prime 2}(\xi)=q(\xi) .
$$

3. Pour la seconde classe d'équations on a

$$
d a-d^{\prime}=0, \quad 2 b(a+c)-2 b^{\prime}+2 b^{2}+h=0, \quad 2 c(a+b)-2 c^{\prime}+2 c^{8}+k=0 .
$$

Il suffit d'indiquer $b, c, d$ si $d \neq 0$ et $b, c, a$ si $d=0$. Je n'indique qu'un type $T$ condensant de nombreux types:

$$
a=\frac{V_{3}^{\prime}-V_{1}^{\prime}}{V_{2}-V_{1}}, \quad b-c=-\frac{3}{2}\left(V_{1}+V_{2}\right), \quad b+c=-\frac{3}{2} \frac{V_{2}^{\prime}-V_{1}^{\prime}}{V_{2}-V_{1}} \quad d=\frac{V_{2}-V_{1}}{2}
$$

$V_{1}$ et $V_{2}$ étant deux intégrales particulières de $V^{\prime \prime}=2 V^{3}+S V+T$, où le second membre désigne soit $2 V^{3}$, soit $2 V^{3}+\alpha V+\beta$, soit $2 V^{3}+X V+\alpha$.

Intégration:

$$
\left.\begin{array}{l}
\frac{Y^{\prime}+2 b}{Y}+\frac{Y^{\prime}-2 c}{\mathrm{I}-Y}=2\left[2 V-V_{1}-V_{2}\right] \\
2 Y-\mathrm{I}=\frac{2 V^{\prime}-V_{1}^{\prime}-V_{2}^{\prime}-a\left[2 V-V_{1}-V_{2}\right]}{2\left(V-V_{1}\right)\left(V-V_{2}\right)}
\end{array}\right\} V^{\prime \prime}=2 V^{3}+S V+T .
$$

4. Pour la dernière catégorie l'une des deux fonctions $b$ ou c est nulle, et l'autre non. A condition de changer s'il le faut $Y$ en $\mathrm{I}-Y$ je suppose $b=0, c \neq 0$. Dans $c e$ cas on a alors $h \neq 0, a+c=\frac{h^{\prime}}{2 h}, 2 a c-2 c^{\prime}+2 c^{2}+k=0, d a-d^{\prime}=0$ l'équation étant

$y^{\prime \prime}=\frac{3}{4}\left(\frac{\mathrm{I}}{y}+\frac{\mathrm{I}}{y-\mathrm{I}}\right)+\left(a-\frac{c}{y-\mathrm{I}}\right) y^{\prime}+y(y-\mathrm{I})\left[4 d^{2}(2 y-\mathrm{I})-\frac{\mathrm{c}^{2}}{(y-\mathrm{I})^{2}}+\frac{h}{y}+\frac{k}{y-\mathrm{I}}\right]$.

On profite d'une substitution $y=Y, X=\varphi(x)$ pour réaliser la condition $2 c+$ $3 a=0$ qui simplifie les résultats et donne les types $T$. On a 3 équations: 
Équations différentielles dont l'intégrale générale est à points critiques fixes.

$I^{0} V_{1}$ désignant une intégrale de $V^{\prime \prime}=2 V^{3}$ on aura

$$
h=2\left(V_{1}^{\prime}+V_{2}^{\prime}\right), \quad c=3 V_{1}^{\prime}, \quad a=-2 V_{1}, \quad d=0, \quad k=6\left(V_{1}^{\prime}-V_{1}^{2}\right) .
$$

Intégration:

$$
Y=\frac{3 T^{2}}{2\left(T^{\prime}+T^{2}-4 V_{1} T+V_{1}^{\prime}+V_{1}^{2}\right)}, \quad T=\frac{V^{\prime}-V_{1}^{\prime}}{\bar{V}-V_{1}}+V+V_{1}, \quad V^{\prime \prime}=2 V^{9} .
$$

$\left.2^{\circ}\right) V_{1}$ désignant une intégrale de $V^{\prime \prime}=2 V^{3}+\alpha V+\beta$ on prend

$$
h=2\left(V_{1}^{\prime}+V_{1}^{\prime}\right)+\alpha, \quad d=\frac{\beta}{h}, a=-\frac{h^{\prime}}{h}, c=\frac{3}{2} \frac{h^{\prime}}{h} .
$$

Intégration:

$$
\begin{gathered}
Y=\frac{3 T^{2}}{2 T^{\prime}+2 T^{2}-\left(4 c+4^{d}+2 a\right) T+h}, \quad T=\frac{V^{\prime}-V_{1}^{\prime}}{V-V_{1}}+V+V_{1}, \\
V^{\prime \prime}=2 V^{s}+\alpha V+\beta .
\end{gathered}
$$

$\left.3^{\circ}\right) V_{1}$ désignant une intégrale de $V^{\prime \prime}=2 V^{3}+X V+\alpha$

$$
h=2\left(V_{1}^{\prime}+V_{1}^{3}\right)+X, \quad d=\frac{\alpha+\frac{\mathrm{I}}{2}}{h^{\prime}}, a=-\frac{h^{\prime}}{h}, \quad c=\frac{3}{2} \frac{h^{\prime}}{h}
$$

même mode d'intégration que précédemment sauf que $V$ satisfait à $V^{\prime \prime}=2 V^{3}+$ $X V+\alpha$.

\section{CHAPITRE V.}

Equations $y^{\prime \prime}=\left[\frac{2}{3 y}+\frac{\mathrm{x}}{2(y-\mathrm{I})}\right] y^{\prime 8}+B(y, x) y^{\prime}+C(y, x)$.

1. On reconnait aisément que l'équation doit être de la forme

$$
\begin{aligned}
& y^{\prime \prime}=\left[\frac{2}{3 y}+\frac{\mathrm{I}}{2(y-1)}\right] y^{\prime 2}+\left(a y+b+\frac{c}{y}\right) y^{\prime}+ \\
& +y(y-\mathrm{I})\left[\frac{3 a^{2}}{8} y+\frac{3}{2}\left(a^{\prime}-a b\right)-\frac{3}{4} a^{2}+\frac{3 c^{2}}{y^{2}}+\frac{h}{(y-1)^{2}}+\frac{6\left(c^{\prime}-b c\right)-3 c^{2}}{2 y}+\frac{h}{3(y-1)}\right] \\
& \text { avec } 2 h(a+b+c)-h^{\prime}=0 .
\end{aligned}
$$


Si l'on fait le changement de fonction

$$
3\left(w+\frac{c}{4}-\frac{a}{5}\right)=\frac{y^{t}+3 c}{y}-\frac{3 a y}{2}
$$

on obtient une équation qui résoud la question

$$
w^{\prime \prime}=-w w^{\prime}+w^{3}+\left(\frac{2 a}{5}+b+\frac{c}{4}\right)\left(w^{2}+3 w^{\prime}\right)+A(x) w+B(x) .
$$

Il y a alors avantage à faire un changement de variables $y=Y, X=\varphi(x)$, $w=W \varphi^{\prime}(x)$ qui conduit à la forme la plus simple pour l'équation en $W$, ̀̀ savoir

$$
W^{\prime \prime}=-W W^{\prime}+W^{3}+A_{1}(X) W+B_{1}(X)
$$

étudiée par M. Painlevé. Je pose, comme l'indique la marche des calculs $q=\frac{3 c}{4}-\frac{3 a}{10}, r=-\frac{3 a}{5}-\frac{3 c}{4}$ et $2 h=-9 s^{2}$. Je prends tout de suite le type réduit $T$ où l'on suppose que $\frac{2 a}{5}+b+\frac{c}{4}=0$ et l'intégration est

$$
\begin{gathered}
Y-\mathrm{I}=\frac{3}{2} \frac{(W-q)^{2}-s^{2}}{\left[W^{\prime}-q^{\prime}+r(W-q)\right]-\left[(W-q)^{9}\right.} \overline{\left.-s^{2}\right]}, \\
W^{\prime \prime}=-W W^{\prime}+W^{3}-\mathrm{I} 2 V_{1} X+\mathrm{I} 2 V_{1}^{\prime} \text { avec } V_{1}^{\prime}=6 V_{1}^{\prime}+S, \quad S=0, \frac{-\mathrm{I}}{24} \text { ou } X
\end{gathered}
$$

les quantités $q, r, s$ étant données par les formules

$$
\begin{gathered}
q=\frac{I}{2}\left(\frac{V_{3}^{\prime}-V_{1}^{\prime}}{V_{3}-V_{1}}+\frac{V_{2}^{\prime}-V_{1}^{\prime}}{V_{3}-V_{1}}\right), s=\frac{I}{2}\left(\frac{V_{3}^{\prime}-V_{1}^{\prime}}{V_{3}-V_{1}}-\frac{V_{2}^{\prime}-V_{1}^{\prime}}{V_{3}-V_{1}}\right), \\
r=-\frac{s^{\prime}}{s}, V_{2} \text { et } V_{3} \text { étant } 2 \text { autres intégrales de } V^{\prime \prime}=6 V^{2}+S .
\end{gathered}
$$

Jo rappelle d'ailleurs que l'équation en $W$ s'intègre en écrivant

$$
W=\frac{V^{\prime}-V_{1}^{\prime}}{V-V_{1}}, \quad V^{\prime \prime}=6 V^{2}+S
$$

2. Dans ces formules il faut se livrer à un calcul de limites si $V_{3}$ tend vers $V_{2}$, quand on suppose $V_{3}$ différent de $V_{1}$ ou quand $V_{3}$ et $V_{2}$ tendent séparément vers $V_{1}$ ou simultanément vers $V_{1}$.

Soit $V(X, \alpha, \beta)$ l'intégrale générale de $V^{\prime \prime}=6 V^{2}+S$, où $\alpha$ et $\beta$ désignent deux constantes arbitraires; si $\alpha=\alpha_{1}, \beta=\beta_{1}$ on a $V_{1}$. Alors si $V_{2}$ tend vers $V_{1}$ 
Équations différentielles dont l'intégrale générale est à points critiques fixes.

on doit remplacer $\frac{V_{2}^{\prime}-V^{\prime}}{V_{2}-V_{1}}$ par $\frac{u^{\prime}}{u}$ où $u=\lambda \frac{\partial V_{1}}{\partial \alpha_{1}}+\mu \frac{\partial V_{1}}{\partial \beta_{1}}$ et $\lambda$ et $\mu$ deux constantes arbitraires; de même si $V_{3}$ tendait vers $V_{1}$ on remplacerait $\frac{V_{3}^{\prime}-V^{\prime}}{V_{3}-V_{1}^{1}}$ par $\frac{v^{\prime}}{v}$ où $v=\lambda^{\prime} \frac{\partial V_{1}}{\partial \alpha_{1}}+\mu^{\prime} \frac{\partial V_{1}}{\partial \beta_{1}}$ où $\lambda^{\prime}$ et $\mu^{\prime}$ sont deux constantes arbitraires.

Ces formules donnent alors complètement la solution si $V_{2}$ et $V_{3}$ sont différentes de $V_{1}$ et différentes entre elles, ou si l'une des quantités $V_{2}$ ou $V_{3}$ a seule tendu vers $V_{1}$ ou si elles sont toutes deux tendu vers $V_{1}$ avec $\lambda \iota^{\prime}-\mu \lambda^{\prime} \neq 0$.

Si $V_{2}$ étant différent de $V_{1}, V_{3}$ tend vers $V_{2}$ il faut donner des explications complémentaires pour $r$; de même si $V_{2}$ a tendu vers $V_{1}$ ainsi que $V_{3}$ et que $\lambda \mu^{\prime}-\mu \lambda^{\prime}=0$, c'est-̀̀-dire $\lambda=\lambda^{\prime}, \iota=\mu^{\prime}$ puisque $\lambda$ et $\mu$ sont simplement déterminés à un facteur près de proportionalité.

Prenons $V_{2} \neq V_{1}$ et $V_{8}$ tendant vers $V_{2}$, alors $q$ tend vers $\frac{V_{2}^{\prime}-V_{1}^{\prime}}{V_{2}-V_{1}}$ et $r$ vers $-\frac{\varrho^{\prime}}{\varrho}$ où l'on calcule $\varrho$ par l'intermédiaire de la quantité $u_{2}=\lambda \frac{\partial V_{2}}{\partial \alpha_{2}}+\iota \frac{\partial V_{2}}{\partial \beta_{2}}$ et par la formule $\varrho=\frac{d}{d x}\left(\frac{u_{2}}{V_{2}-V_{1}}\right)$.

Si $V_{2}$ et $V_{3}$ ont tendu toutes les deux vers $V_{1}$ et si $\lambda^{\prime}=\lambda, \mu^{\prime}=\mu$ on a $s=0, q=\frac{u^{\prime}}{u}$ où $u=\lambda \frac{\partial V_{1}}{\partial \alpha_{1}}+\mu \frac{\partial V_{1}}{\partial \beta_{1}}$ et $r=-\frac{\varrho^{\prime}}{\varrho}$ en posant cette fois

$$
\begin{aligned}
& p=\lambda^{2} \frac{\partial^{2} V_{1}}{\partial \alpha_{1}^{2}}+2 \lambda_{1} \mu \frac{\partial^{2} V_{1}}{\partial \alpha_{1} \partial \beta_{1}}+\iota^{2} \frac{\partial^{2} V_{1}}{\partial \beta_{1}^{2}} \\
& \varrho=\lambda^{\prime} \frac{d}{d x}\left(\frac{p}{u}\right)+\frac{\mu^{\prime}}{u^{z}} .
\end{aligned}
$$

Cet exemple montre combien doit être minutieuse la formation de tous les types $T$ puisqu'il faut considérer 3 integrales $V_{1}, V_{2}, V_{3}$ de la même équation $V^{\prime \prime}=6 V^{2}+S$. Si je prends $S=0$ j'ai par exemple les intégrales de $V^{\prime \prime}=6 V^{2}$ qui sont ou o ou $\frac{\mathrm{I}}{\left(X-X_{v}\right)^{2}}$ ou $\alpha^{2} p(\alpha X+\beta, o$, I $)$ ce qui fait en tout pour $V_{1}, V_{2}, V_{3}$ un nombre considérable de combinaisons. 


\section{CHAPITRE VI.}

Equations $y^{\prime \prime}=\frac{y^{\prime 2}}{2}\left(\frac{\mathrm{I}}{y}+\frac{\mathrm{I}}{y-\mathrm{I}}+\frac{\mathrm{I}}{y-H}\right)+B(y, x) y^{\prime}+C(y, x)$.

1. - Si l'on prend $H=\alpha$ où $\alpha$ est une constante, on obtient seulement deux équations dont les formes $T$ sont

(I) $Y^{\prime \prime}=\frac{Y^{\prime 2}}{2}\left(\frac{\mathrm{I}}{Y}+\frac{\mathrm{I}}{Y-\mathrm{I}}+\frac{\mathrm{I}}{Y-\alpha}\right)$

(2) $Y^{\prime \prime}=\frac{Y^{\prime 2}}{2}\left(\frac{\mathrm{I}}{\bar{Y}}+\frac{\mathrm{I}}{Y-\mathrm{I}}+\frac{\mathrm{I}}{Y-\alpha}\right)+Y^{\prime}(Y-\mathrm{I})(Y-\alpha)\left[\beta+\frac{\gamma}{Y^{2}}+\frac{\delta}{(Y-\mathrm{I})^{2}}+\frac{\varepsilon}{(Y-\alpha)^{2}}\right]$

et les formes $\Theta$ correspondantes

(I) $\eta^{\prime \prime}=\frac{\eta^{\prime 2}}{2}\left(\frac{\mathrm{I}}{\eta}+\frac{\mathrm{I}}{\eta-\mathrm{I}}+\frac{\mathrm{I}}{\eta-\alpha}\right)+q(\xi) \eta^{\prime}$

(2') $\eta^{\prime \prime}=\frac{\eta^{\prime 2}}{2}\left(\frac{\mathrm{I}}{\eta}+\frac{\mathrm{I}}{\eta-\mathrm{I}}+\frac{\mathrm{I}}{\eta-\alpha}\right)+$

$$
+\frac{q^{\prime}(\xi)}{2 q(\xi)} \eta^{\prime}+q(\xi) \eta(\eta-\mathrm{I})(\eta-\alpha)\left[\beta+\frac{\gamma}{\gamma^{2}}+\frac{\delta}{(\eta-\mathrm{I})^{2}}+\frac{\varepsilon}{(\eta-\alpha)^{2}}\right] .
$$

On passe du type $\Theta$ au type $T$ par $\eta_{i}=Y, X=\varphi(\xi)$ où $\varphi$ est donné pour

(I) par 2 quadratures $\frac{\varphi^{\prime \prime}}{\varphi^{\prime}}=q(\xi)$ et pour la seconde par une seule $\varphi^{\prime 8}=q(\xi)$.

L'intégrale de (I) s'obtient en écrivant $Y^{\prime 2}=K Y(Y-\mathrm{I})(Y-\alpha)$ et celle de (2) en écrivant $Y^{\prime 2}=\left[2 \beta Y-\frac{2 \gamma}{Y}-\frac{2 \delta}{Y-\mathrm{x}}-\frac{2 \varepsilon}{Y-\alpha}+K\right] Y(Y-\mathrm{I})(Y-\alpha)$.

2. - En supposant $H=X$ on obtient le type irréductible VI qui est

$$
\begin{aligned}
Y^{\prime \prime}=\frac{Y^{\prime / 2}}{2}\left(\frac{\mathrm{I}}{Y}+\frac{\mathrm{I}}{Y-\mathrm{I}}+\frac{\mathrm{I}}{Y-X}\right)-Y^{\prime}\left(\frac{\mathrm{I}}{Y-X}+\frac{\mathrm{I}}{X}+\frac{\mathrm{I}}{X-\mathrm{I}}\right)+ \\
\quad+\frac{Y(Y-\mathrm{I})(Y-X)}{2 X^{2}(X-\mathrm{I})^{2}}\left[\alpha-\beta \frac{X}{Y^{2}}+\gamma \frac{(X-\mathrm{I})}{(Y-\mathrm{I})^{8}}-\frac{(\delta-\mathrm{I}) X(X-\mathrm{I})}{(Y-X)^{2}}\right] .
\end{aligned}
$$

Les substitutions $Y Y_{1}=\mathrm{I}, X X_{1}=\mathrm{I}$ ou $Y+Y_{1}=\mathrm{I}, X+X_{1}=\mathrm{I}$ on $Y=X Y_{1}$, $X X_{1}=\mathrm{I}$ ne changent pas la forme de l'équation et permutent entre elles les constantes $\alpha, \beta, \gamma, \delta$.

Pour $\alpha, \beta, \gamma, \delta$ nuls ensemble, M. Painlevé a donné l'intégration de cette équation: pour $\alpha, \beta, \gamma, \delta$ quelconques cette équation est irréductible. - 


\section{CHAPITRE VII.}

1. - Je prends ici l'exemple particulier (chapitre I, paragraphe 8)

$$
y^{\prime \prime}=\frac{y^{\prime 2}}{2 y}+b y^{\prime}+d y^{3}+e y^{2}+f y+\frac{h}{y}
$$

Je vais chercher la condition nécessaire pour que l'intégrale générale admette des pôles mobiles et montrer que cette condition est nécessaire pour que l'intégrale générale soit à points critiques fixes. Lc mode de calcul s'étendra évidemment à tous les autres cas et légitimera le résultat général énoncé dans le préface, paragraphe 7 .

Pour simplifier les calculs faisons, si besoin en est, $b=0, d=\frac{3}{2}$ par une substitution $y=\lambda(x) Y, X=\varphi(x)$. Soit done

$$
y^{\prime \prime}=\frac{y^{\prime 2}}{2 y}+\frac{3 y^{3}}{2}+e y^{2}+f y+\frac{h}{y}
$$

Il y a deux familles de pôles simples

$$
y=\frac{\varepsilon}{x-x_{0}}+B+C\left(x-x_{0}\right)+D\left(x-x_{0}\right)^{2}+\cdots
$$

où $\varepsilon=+\mathrm{I}$ ou $-\mathrm{I}, B=-\frac{e_{0}}{4}, C=\frac{\varepsilon e_{0}^{2}}{\mathrm{I} 6}-\frac{e_{0}^{\prime}}{3}-\frac{f_{0} \varepsilon}{3}$ et $D$ est arbitraire, il disparait en effet de la relation obtenue en égalant les termes constants dans les 2 membres, mais cette relation entraîne pour avoir un tel pôle $\frac{e_{0}^{\prime \prime}}{2}+\varepsilon\left(f_{0}^{\prime}-\frac{e_{0} e_{0}^{\prime}}{4}\right)=0$, ce qui entraîne puisque $x_{0}$ est quelconque et que $\varepsilon$ peut être + I ou $-\mathbf{I} e \equiv o$ $f^{\prime} \equiv \frac{e e^{\prime}}{4}$. Ecrivons alors

(4)

$$
\left\{\begin{array}{l}
y=\frac{\varepsilon}{x-x_{0}}+B+C\left(x-x_{0}\right)+\lambda\left(x-x_{0}\right)^{2}+\cdots=\frac{\mathrm{I}}{z} \\
y^{\prime}=\frac{-\varepsilon}{\left(x-x_{0}\right)^{2}}+C+2 \lambda\left(x-x_{0}\right)+\cdots
\end{array}\right.
$$

ou $B$ et $C$ ont les valeurs indiquées. De la première équation on tire aisément les développements en série pour $x-x_{0}$ et $y^{\prime}$ 
(5)

$$
\left\{\begin{array}{l}
x-x_{0}=\varepsilon z\left\{I-\frac{e_{0}}{4} z+\left(\frac{e_{0}^{2}}{8}-\frac{\varepsilon e_{0}^{\prime}}{3}-\frac{f_{0}}{3}\right) z^{2}+\cdots\right\} \\
y \quad=\frac{-\varepsilon}{z^{2}}-\frac{\varepsilon e_{0}}{2 z}+\left(\frac{\varepsilon e_{0}^{2}}{8}-e_{0}^{\prime}-f_{0} \varepsilon\right)+4 \lambda \varepsilon z+\cdots
\end{array}\right.
$$

J'applique maintenant la formule

d'où

$$
\varphi\left(x_{0}\right)=\varphi\left(x+x_{0}-x\right)=\varphi(x)-\frac{x-x_{0}}{\mathrm{I}} \varphi^{\prime}(x)+\frac{\left(x-x_{0}\right)^{2}}{\mathrm{I}, 2} \varphi^{\prime \prime}(x) \cdots
$$

$$
\begin{aligned}
e_{0} & =e-\left(x-x_{0}\right) e^{\prime}+\frac{\left(x-x_{0}\right)^{2}}{\mathrm{I} \cdot 2} e^{\prime \prime} \cdots \\
\frac{e_{0}}{z} & =\frac{e}{z}-\varepsilon e^{\prime}+z\left(\frac{e^{\prime \prime}}{2}+\frac{\varepsilon e e^{\prime}}{4}\right)+\cdots \\
\frac{\varepsilon e_{0}^{2}}{8}-e_{0}^{\prime}-f_{0} \varepsilon & =\frac{\varepsilon e^{2}}{8}-e^{\prime}-f \varepsilon-\left(\alpha-x_{0}\right)\left[\frac{\varepsilon e e^{\prime}}{4}-e^{\prime \prime}-f^{\prime} \varepsilon\right]+\cdots \\
& =\frac{\varepsilon e^{2}}{8}-e^{\prime}-f \varepsilon-\varepsilon z\left[\frac{\varepsilon e e^{\prime}}{4}-e^{\prime \prime}-f^{\prime} \varepsilon\right]+\cdots
\end{aligned}
$$

J'ai finalement

(6)

$$
\left\{\begin{array}{l}
y^{\prime}=-\frac{\varepsilon}{z^{2}}-\frac{\varepsilon e}{2 z}+\left(\frac{\varepsilon e^{8}}{8}-\frac{e^{\dagger}}{2}-f \varepsilon\right)+\varepsilon z\left[4 \lambda-\frac{3 \varepsilon e e^{t}}{8}+\frac{3 e^{\prime \prime}}{4}+f^{\prime} \varepsilon\right]+\cdots \\
y=\frac{I}{z}
\end{array}\right.
$$

Le calcul ainsi préparé conduit alors à prendre comme inconnues les fonctions $u$ et $z$ définies par

(7)

$$
\left\{\begin{array}{l}
y=\frac{\mathbf{I}}{z} \\
y^{\prime}=\frac{-\varepsilon}{z^{2}}-\frac{\varepsilon e^{2}}{2 z}+\left(\frac{\varepsilon e^{2}}{8}-\frac{e^{\prime}}{2}-f \varepsilon\right)+u z .
\end{array}\right.
$$

Ceci conduit au système

$$
\left\{\begin{array}{l}
z^{\prime}=\varepsilon+z \varphi(u, z) \\
u^{\prime}=\left[\frac{e^{\prime \prime}}{2}+\varepsilon\left(f^{\prime}-\frac{e e^{\prime}}{4}\right)\right] \frac{\mathrm{I}}{z}+\psi(u, z),
\end{array}\right.
$$

où $\psi$ et $\varphi$ sont deux polynômes en $u$ et $z$. Faisons maintenant dans le système (8) équivalent à (2) la substitution $x==x_{0}+\alpha X, z=\alpha Z, u=U$; le nouveau 
systeme a ses points critiques fixes comme (8) pour toute valeur non nulle de $\alpha$, donc encore pour $\alpha=0$. Or pour $\alpha=0$ il se réduit à

$$
\left\{\begin{array}{l}
Z^{\prime}=\varepsilon \\
U^{\prime}=\left\{\frac{e_{0}^{\prime \prime}}{2}+\varepsilon\left(f_{0}^{\prime}-\frac{e_{0} e_{0}^{\prime}}{4}\right)\right\} \frac{\mathrm{I}}{Z}
\end{array}\right.
$$

qui s'intègrc évidemment par un logarithme si $\frac{e_{0}^{\prime \prime}}{2}+\varepsilon\left(f_{0}^{\prime}-\frac{e_{0} e_{0}^{\prime}}{4}\right)$ n'est pas nul. On retrouve ainsi les résultats annoncés.

Equations

\section{CHAPITRE VIII.}

$$
y^{\prime \prime}=\left(\mathrm{I}-\frac{\mathrm{I}}{n}\right) \frac{y^{\prime 2}}{y}+\left(a y+b+\frac{c}{y}\right) y^{\prime}-\frac{n a^{3}}{(n+2)^{2}} y^{3}+e y^{8}+f y+g+\frac{h}{y}
$$

avec $a \equiv$ o ou $a \equiv$ 非 et $(n-2)^{2} h+n c^{2} \equiv$ o.

1. - Je suppose d'abord $n=2$, alors je suppose $a h \equiv=0$, pour ne pas étudier de nouveau des équations rencontrées ailleurs. On a alors $c=0, b=\frac{h^{\prime}}{2 h}$, $2 e+a b-a^{\prime}=0, g=0$. On peut par $y=\lambda(x) Y, X=\varphi(x)$ supposer $a=-2$, $h=-\frac{\mathrm{I}}{2}$ d'où

$$
y^{\prime \prime}=\frac{y^{\prime 8}}{2 y}-2 y y^{\prime}-\frac{y^{3}}{2}+f y-\frac{\mathrm{I}}{2 y}
$$

Intégration :

$$
y=\frac{z^{\prime}}{z}, \quad z^{\prime \prime \prime} z^{\prime}=\frac{z^{\prime \prime 8}}{2}+f z^{\prime 8}-\frac{z^{9}}{2}
$$

Je dérive, d'où

$$
\boldsymbol{z}^{\prime \prime \prime \prime}=2 f \boldsymbol{z}^{\prime \prime}+f^{\prime} \boldsymbol{z}^{\prime}-\boldsymbol{z}
$$

On est ramené à une équation linéaire du $4^{\circ}$ ordre; si on appelle $z_{1}, z_{2}, z_{s}, z_{\jmath}$ 4 solutions linéairement indépendantes de (2) on pourra écrire que l'intégrale de (2), puisqu'elle vérifie (3) est de la forme 


$$
z=C_{1} z_{1}+C_{2} z_{2}+C_{3} z_{3}+C_{4} z_{4}
$$

et en substituant dans (2) nous obtenons une relation entre les $C$ de la forme $\Sigma A_{i j} C_{i j}=$ o, où les $A_{i j}$ sont des constantes, $i$ et $j$ étant deux entiers différents égaux à $\mathrm{I}, 2,3$ ou 4 . On voit donc que $y$ s'exprime rationnellement au moyen de 3 constantes arbitraires.

L'équation (I) pourra si l'on veut être prise comme type $T$, le type $\Theta$ pourra être pris sous la forme

$$
\eta^{\prime \prime}=\frac{\eta^{\prime 2}}{2 \eta}-2 \eta \eta^{\prime}+\frac{h^{\prime}}{2 h} \eta_{i}^{\prime}-\frac{\eta^{3}}{2}+\frac{h^{\prime}}{2 h} \eta^{2}+f \eta+\frac{h}{\eta}
$$

et on l'intègre de la même façon. -

2. - Supposons $n \neq 2, c \neq 0$. Si $a \equiv 0$, on prend $e \equiv$ o (pour $n=4$, on peut supposer $e=\equiv$, j'ai étudié autre part ce cas). - - Si $a \equiv \equiv$ il y a deux familles de pôles simples mobiles, un $y=-\frac{n+2}{n a_{0}} \frac{I}{x-x_{0}}+\cdots$ qui donne la relation $(n+2) e+n\left(a b-a^{\prime}\right)=0$ et un autre

$$
y=-\frac{(n+\mathrm{I})(n+2)}{n a_{0}} \frac{\mathrm{I}}{x-x_{0}}+a_{1}+\frac{a_{2}}{x-x_{0}}+\cdots+a_{p}\left(x-x_{0}\right)^{p-1}+\cdots
$$

qui ne donne pas de relation: pour le voir supposons $a \equiv-\frac{n+2}{n}$, on voit aisément que l'on a en égalant les termes en $\left(x-x_{0}\right)^{p-3},(p+\mathrm{I})(p+n+\mathrm{I}) a_{p}=$ $=\varphi\left(a_{p-1}, a_{p-2}, \ldots a_{1}\right)$ donc le multiplicateur de $a_{p}$ n'est jamais nul, car $n$ est un entier positif.

Si l'on veut s'occuper des zéros, on applique ce qui précède en changeant $y$ en $\frac{I}{y_{1}}$ et appelant $-n=n^{\prime}$ d'où l'équation

$$
y_{1}^{\prime \prime}=\left(\mathrm{I}-\frac{\mathrm{I}}{n^{\prime}}\right) \frac{y_{1}^{\prime 2}}{y_{1}}+c y_{1} y_{1}^{\prime}+b y_{1}^{\prime}+a \frac{y_{1}^{\prime}}{y_{1}}-\frac{n^{\prime} c^{2}}{\left(n^{\prime}+2\right)^{2}} y_{1}^{8}-g y_{1}^{2}-f y_{1}-e-\frac{n^{\prime} a}{\left(n^{\prime}-2\right)^{2}} \frac{\mathrm{I}}{y_{1}} .
$$

Mais alors cette fois les deux zéros de $y$ donnent chacun une relation, l'une est $(n-2) g-n\left(c b-c^{\prime}\right)=0$ et l'autre est d'autant plus compliquée à écrire que $n$ est plus grand. Si nous ne tenons pas compte de cette relation j'ai une équation qui est intégrable, et qui sera à points critiques fixes quand cette relation non écrite sera satisfaite. Ecrivons en effet, (nous supposerons $c=-\frac{n-2}{n}$ ) 
(5) $y^{\prime \prime}=\left(\mathrm{x}-\frac{\mathrm{I}}{n}\right) \frac{y^{\prime 8}}{y}+a y y^{\prime}+b y^{\prime}-\frac{n-2}{n} \frac{y^{\prime}}{y}-\frac{n a^{2}}{(n+2)^{8}} y^{3}+\frac{n\left(a^{\prime}-a b\right)}{n+2} y^{2}+\left(y-b-\frac{\mathrm{I}}{n y}\right.$.

Je pose

$$
y^{\prime}-\mathbf{I}-\frac{a n y^{2}}{n+2}=n u y
$$

ce qui conduit au système

$$
\left\{\begin{array}{l}
u^{\prime}+u^{2}-b u-\frac{2 a}{n(n+2)}-\frac{f}{n}=0 \\
y^{\prime}-\frac{a n y^{2}}{n+2}-n u y-\mathrm{I}=0 .
\end{array}\right.
$$

Si $a \equiv$ 非, j'ai deux équations de Riccati, si $a \equiv 0$ j'ai une équation de Riccati suivie d'une équation linéaire. Si $a \equiv 0$, jo pose $u=\frac{v^{\prime}}{v}$ et en appelant $v_{1}$ et $v_{2}$ deux solutions de $v^{i}=b v^{\prime}+\frac{f v}{n}$, on a

$$
y=\left(C_{1} v_{1}+C_{2} v_{2}\right)^{n}\left[\frac{\mathrm{I}}{C_{z}^{n}}+\int \frac{d n}{\left(C_{1} v_{1}+C_{2} v_{2}\right)^{n}}\right]
$$

Il n'y a qu'à exprimer que $v$ étant solution de $v^{\prime \prime}=b v^{\prime}+\frac{f v}{n}$ définie par $v_{0}=0, v_{0}^{\prime}$ arbitraire pour $x=x_{0}$, la quantité $\frac{\mathrm{I}}{v^{n}}$ a pour $x=x_{0}$ son résidu nul, sinon $y$ a une singularité logarithmique. Si $a=\sum^{\prime}=0$ on pose encore $u=\frac{v^{\prime}}{v}$ et $y=-\frac{n+2}{n a} \frac{z^{\prime}}{z}$ d'où

$$
\begin{gathered}
v^{\prime \prime}=b v^{\prime}+\left(\frac{2 a}{n(n+2)}+\frac{f}{n}\right) v \\
z^{\prime \prime}-\left(\frac{a^{\prime}}{a}+n \frac{v^{\prime}}{v}\right) z^{\prime}+\frac{n a}{n+2} z=0 .
\end{gathered}
$$

Il n'y a qu'à appliquer les résultats de Fuchs à l'équation en $z$ pour voir qu'à un zéro de $v$ correspond pour $z$ un point logarithmique (les racines de l'équation caractéristique sont en effet o et $n+\mathrm{I}$ ), à moins qu'une certaine condition ne soit remplie.

On retrouve ainsi la relation que nous avions formée plus haut par un autre moyen.

Cette méthode a un inconvénient, c'est de ne pas donner les coefficients de l'équation (5) explicitement. J'y arrive par un autre procédé. 
3. - J'écris

(9)

$$
\zeta=\frac{(n-\mathrm{I}) y^{\prime}+\mathrm{I}}{n y}-\frac{a(n-\mathrm{I}) y}{n+2}
$$

d'où

$$
y\left[\zeta^{\prime}+\zeta^{2}-\frac{a\left(2 n-2-n^{2}\right)}{n(n+2)}-\frac{n-1}{n} f-b \zeta\right]+b+\frac{n-2}{n-1} \zeta=0 .
$$

On remarque que si $n=2$ l'opération est terminée, car alors $b=0$ et je retrouve deux équations de Riccati. Sinon je pose

$$
\left\{\begin{array}{l}
b+\frac{n-2}{n-\mathrm{I}} \zeta=\frac{n-2}{n-\mathrm{I}} z, \quad \alpha=\frac{-n b}{n-2} \\
\beta=-\frac{n-\mathrm{I}}{n-2} b^{\prime}+\frac{(n-\mathrm{I})^{2}}{(n-2)^{2}} b^{2}-\frac{a\left(2 n-2-n^{2}\right)}{n(n+2)}-\frac{n-\mathrm{I}}{n} f .
\end{array}\right.
$$

J'ai exprimé $y$ en $\zeta$, done en $z$ et j'ai

$$
z^{\prime \prime}=\frac{z^{\prime 2}}{z}\left[\mathrm{I}-\frac{\mathrm{I}}{n-2}\right]-\frac{n}{n-2} z z^{\prime}-\frac{\alpha n}{n-2} z^{\prime}+\frac{\beta(n-4)}{n-2} \frac{z^{\prime}}{z}-\frac{z^{3}}{n-2}-\frac{\alpha n}{n-2} z^{8}
$$

$$
-\left[\alpha^{\prime}+\alpha^{2} \frac{n-\mathrm{I}}{n-2}+\beta \frac{n^{8}-2 n+2}{(n-\mathrm{I})(n-2)}-\frac{a n(n-2)}{(n+2)(n-\mathrm{I})}\right] z-\left[\frac{\alpha \beta n}{n-2}+\beta^{\prime}\right]-\frac{\beta^{9}}{(n-2) z} .
$$

Si $\beta \equiv 0$, j'ai précisément une équation étudiée (chapitre ${ }^{8 r}$, deuxième type), dont l'intégrale est à points critiques fixes et contient rationnellement les constantes d'intégration: on sait que dans ce cas on a une équation linéaire du socond ordre à résoudre et $(n-I)$ quadratures à faire ensuite. Si $\beta \equiv 0$, on remarque que les coefficients de (5) sont exprimés explicitement au moyen de deux fonctions arbitraires $a, b$ et de leurs dérivées. On remarque d'ailleurs que pour $n=3, \beta$ est nécessairement nul. ${ }^{1}$

Si $\beta \equiv \Sigma_{1} 0$, on a une équation du même type (où le coefficient de $z^{3}$ n'est pas nul) mais où $n$ a été remplacé par $n-2$. Il n'y a plus qu'à recommencer sur elle. Si $n=4$, cette équation serait justement celle étudiée au premier paragraphe de ce chapitre. Sinon on recommence l'application de la méthode.

Conclusion: si $n$ est impair, ou bien $\beta \equiv 0$ et alors l'application faite une fois de la méthode suffit, ou bien $\beta \equiv 0$, alors on recommencera l'application et on arrivera au bout d'un certain nombre de fois à une équation où cette quantité $\beta$ sera nulle. Donc pour $n=2 p+I$, j'ai $p$ types distincts suivant qu'il faut appliquer la méthode $\mathrm{I}, 2 \ldots, p$ fois.

$$
1 \text { Pour } n=3 \text { j'ai } z^{\prime \prime}=-3 z z^{\prime}-z^{8}-3 a\left(z^{\prime}+z^{2}\right)-\left[\iota^{\prime}+2 \alpha^{2}-\frac{3 a}{10}\right] z \text {; je pose } z=\frac{t^{\prime}}{t} \text { d'où }
$$
$t^{\prime \prime \prime}+3 \alpha t^{\prime \prime}+\left[\alpha^{\prime}+2 a^{2}-\frac{j a}{10}\right] t^{\prime}=0$, c'est bien une équation linéaire du second ordre suivie d'une quadrature. 
Équations différentielles dont l'intégrale générale est à points critiques fixes.

Si $n$ est pair, ou bien $\beta$ est nul pour l'équation et alors l'application de la méthode faite une fois ramène à un type connu (chapitre $I^{\mathrm{er}}$, deuxième type), ou bien il sera nul pour l'une des équations suivantes, ce qui ramène toujours à ce type, ou bien on arrivera à une équation du type étudié au ${ }^{{ }^{\text {or }}}$ paragraphe de ce chapitre: donc pour $n=2 p$, il $\mathrm{y}$ a $p$ types distincts.

Il n'y a plus qu'à montrer que les coefficients de (5) peuvent être écrits explicitement, quel que soit $n$ au moyen de deux fonctions arbitraires et de leurs dérivées d'ordre $n-2$ au plus.

Si $\beta \equiv 0$, c'est déjà démontré. Si $\beta \equiv 0$, supposons que pour les valeurs numériques $2,3, \ldots n-2$ on sache obtenir ce résultat pour les équations étudiées: dans (I2) le terme en $\frac{z^{\prime}}{z}$ me donnera $\alpha$ ou $b$; le terme en $z^{\prime}$ me donne $\beta$, celui en $z$ me donne $a$, donc en rapprochant ces 2 résultats, j'ai $f$; j'ai donc obtenu $a, b, f$; c'est un résultat vrai pour $n=2, n=3$ done il s'étend à toutes les valeurs positives de $n$. 